\title{
Völkerrechtliche Regelungen von Staatsangehörigkeitsfragen im Dekolonialisierungsprozeß
}

\author{
Von Hellmuth Hecker
}

\section{Einleitung}

Der Dekolonialisierungsprozeß, der nach dem Zweiten Weltkrieg begann und der auch eine Neuorientierung der Beziehungen der ehemaligen Kolonialmächte zu den unabhängig gewordenen Besitzungen und auch dieser untereinander beinhaltet, hat sich auch auf das Staatsangehörigkeitsrecht ausgewirkt. Zahlreiche vertragliche Regelungen in den verschiedensten Zusammenhängen sind hier getroffen worden, bei und nach Erlangung der Unabhängigkeit. Dabei schälen sich innerhalb der verschiedenen Rechtskreise Gemeinsamkeiten heraus, deren Rechtsnatur nur aus einer allumfassenden rechtsvergleichenden Darstellung durchsichtig wird. Um allgemeine Rechtsgrundsätze, Gewohnheitsrecht oder auch nur Entwicklungstendenzen im Staatsangehörigkeitsrecht nachzuweisen, müssen alle entsprechenden völkerrechtlichen Verträge herangezogen werden, auch die der Industriestaaten untereinander, zumal die Kolonialmächte unter ihnen in ihrer völkerrechtlichen Praxis gegenüber den Entwicklungsländern von ihrer allgemeinen Einstellung zu Staatsangehörigkeitsfragen geprägt wurden. Die Staatsangehörigkeitsregelungen der Industriestaaten unter sich, der Entwicklungsländer unter sich und zwischen beiden können nicht isoliert betrachtet werden, sondern erfordern eine Gesamtschau.

Die völkerrechtlichen Verträge, die Bestimmungen über Staatsangehörigkeitsfragen enthalten, sind bisher noch nicht systematisch und umfassend bearbeitet worden. Lediglich die Abkommen der deutschen Länder und des Deutschen Reiches, ${ }^{1}$ der USA ${ }^{2}$ und Großbritanniens ${ }^{3}$ wurden wissenschaftlich aufbereitet. Dagegen fehlen noch völlig Arbeiten über die Staatsangehörigkeitsverträge Frankreichs, ${ }^{4}$ Italiens ${ }^{5}$ und Spaniens, ${ }^{6}$ welche der

1 H. Hecker, Die Regelung von Staatsangehörigkeitsfragen in völkerrechtlichen Verträgen deutscher Staaten in Vergangenheit und Gegenwart, in: Recht im Dienst des Friedens, Festschrift f. Eberhard Menzel, Berlin 1975 , S. 177-212.

2 H. Hecker u. K. Krakau, Die völkerrechtlichen Verträge der Vereinigten Staaten von Amerika über Fragen der Staatsangehörigkeit einschl. Einbürgerung und Wehrpflicht, in: VRU 1971, S. 69-104.

3 H. Giese, Staatsangehörigkeitsrechtliche Bestimmungen in völkerrechtlichen Vereinbarungen Großbritanniens, Frankfurt 1976, 290 S. (Veröff. a. d. Inst. f. Int. Ang. Bd. 4). Dabei handelt es sich um eine Hamburger Dissertation. Ferner afrik. Verträge 1854-1947: SGS Bd. 38, 481-83.

4 Eine Ubersicht aller StA-Verträge Frankreichs von 1797-1963, in: Hecker/Tomson, Das Staatsangehörigkeitsrecht Frankreichs, Frankfurt 1968, S. 263-282 (SGS Bd. 29). Ferner Verträge Frankreichs mit afrika. Staaten: 1880-1962 SGS Bd. 39, S. 38-40. Darstellung gegenüber 31 Staaten: SGS 29, S. 104-114.

5 Ubersicht aller StA-Verträge Italiens von 1859-1964: In Doerner/Hecker, Das Staatsangehörigkeitsrecht Italiens, Ffm. 1967, S. 344-366 (SGS Bd. 27). Ferner Verträge Italiens mit af rik. Staaten 1880-1950: SGS Bd. 39, S. 40-41. Eine kurze Darstellung der Vertragsinhalte gegenüber 26 Staaten: SGS Bd. 27, S. 131-138.

6 Ubersicht aller StA-Verträge Spaniens 1819-1969: Werkhefte Bd. 25, S. 516-524. Uber die Doppelstaaterverträge Spaniens s. u. 
Zahl und Bedeutung nach ebenso gewichtig sind wie die Verträge der erstgenannten drei Gebiete. Da eine systematische Bearbeitung aller Staatsangehörigkeitsverträge - sie begannen vor etwa 200 Jahren und bezogen sich auch bald schon auf Kolonien - zu umfangreich wäre, beschränkt sich die folgende Darstellung auf die Verträge ab 1945, weil dies Schicksalsjahr des Weltkriegsendes auch eine allgemeine Wende hinsichtlich des Staatsangehörigkeitsrechts beinhaltet. Zunehmende multilaterale Staatsangehörigkeitsverträge, wachsende Bedeutung der Doppelstaaterproblematik und fast völliger Verzicht auf friedensvertragliche Regelung der Staatsangehörigkeit sind einige der hervorstechendsten Merkmale der modernen Vertragspraxis. Angesichts der Tatsache, daß einzig der Friedensvertrag mit Italien von 1947 Staatsangehörigkeitsbestimmungen enthält, nicht dagegen die anderen Friedensverträge und vor allem angesichts des uns besonders angehenden Mangels jeder vertraglichen Regelung der deutschen Staatsangehörigkeit, ${ }^{7}$ ist es allzu verständlich, daß die Ansicht sich verbreiten konnte, nach 1945 habe die Staatsangehörigkeit in völkerrechtlichen Verträgen im Gegensatz zu der Zeit nach Beendigung des Ersten Weltkrieges kaum noch eine Rolle gespielt. Daß diese Ansicht falsch ist, zeigt die in der folgenden Untersuchung herangezogene Zahl von 23 multi- und über 200 bi-lateralen Verträgen. Die Gesamtheit dieser Verträge aus 40 Jahren ließ sich in 5 Komplexe gliedern. Dabei wurden, um den Umfang nicht uferlos anschwellen zu lassen, die Verträge nur mit einer laufenden Nummer gekennzeichnet, die auf eine chronologische Ubersicht am Ende verweist. Ferner wurde darauf verzichtet, die Fundstelle der Verträge im einzelnen anzugeben, da hierfür auf Vorarbeiten verwiesen werden konnte. An Hand der folgenden Abhandlung schält sich heraus, welche Staatsangehörigkeitsprobleme überhaupt von den Staaten für regelungsbedürftig gehalten wurden. Dabei zeigt sich einmal die Vielfalt der Fälle und zum anderen doch eine gewisse Gruppierung von Lösungsmöglichkeiten. Auf jeden Fall bietet die hier folgende Aufbereitung erstmals eine zuverlässige Grundlage für rechtsvergleichende Untersuchungen, für Spezialstudien einzelner Probleme und für Aspekte de lege ferenda. Auch die enge Beziehung von innerstaatlichem Staatsangehörigkeitsrecht und völkerrechtlichen Verträgen zu diesem Thema erscheint dann in hellerem Lichte.

7 Gegenüber den Millionen Deutschen, deren StA nach dem Zweiten Weltkrieg ungeregelt blieb, muten die Regelungen gegenüber den Niederlanden für 23 Personen und Belgien für 60 Personen (Nr. 106 und 74) recht seltsam an. 


\section{A. Doppelstaater ${ }^{8}$}

\section{Allgemeine Rechtsstellung}

In der Haager StA-Konvention von 1930 ist der allgemeine Grundsatz aufgenommen worden, daß Doppelstaater im jeweils anderen Staat keinen diplomatischen Schutz genießen (Art. 4) und in Drittstaaten nur nach ihrer effektiven StA zu behandeln sind (Art. 5). Dieser Grundsatz des Art. 4 wird im Vertrag Nr. 108 wiederholt, sobald der Wohnsitz 6 Monate besteht: vorher ist also abweichend von Art. 4 noch dipl. Schutz möglich. Ebenfalls in Nr. 158: keine Auslieferung von Doppelstaatern, wobei über den Besitz der StA des Wohnsitzstaates allein dieser entscheidet.

Ausnahmen von diesem Prinzip gab es in Art. 13 des durch Nr. 2 aufgehobenen Vertrags v. 1896: Doppelstaater (auch Schutzgenossen) wurden mit 2 Ausnahmen in Italien als Tunesier und in Tunesien als Italiener behandelt. Neuerdings haben die USA in Verträgen mit kommunistischen Staaten ebenfalls eine Ausnahmeregelung getroffen: Doppelstaater, die mit gültigem Visum nach Jugoslawien, Polen, Bulgarien, der DDR oder China einreisen, dürfen dort allein nach ihrer US-StA behandelt werden - und umgekehrt; dies gilt auch, wenn die freiwillige Abreise durch Rechts- oder Verwaltungsmaßnahmen verhindert wurde (Nr. 39, 160, 167, 190, 192). Eine ähnliche Regelung haben die DDR und Österreich getroffen (Nr. 173a). Ein weiterer Fall dieser Ausnahme ist Nr. 154: Falkländer, die jeweils von Großbritannien und Argentinien als ihre StA'en beansprucht wurden, erhielten unabhängig von diesem Anspruch Reisedokumente für Argentinien, das solange darauf verzichtete, sie praktisch als seine StA'en zu beanspruchen.

\section{Wehrpflicht ${ }^{9}$}

\section{Freie Wahl des Wehrdienststaates}

In vielen Verträgen über den Wehrdienst von Doppelstaatern ist ein solches Wahlrecht für alle Fälle vereinbart worden, so in 4 Verträgen Italiens (Nr. 54, 60, 71, 96) und 5 Verträgen Frankreichs $(29,31,32,35,196)$. Nr. 196 fügt noch hinzu: Wird nicht rechtzeitig gewählt, so gilt der Wohnsitz; das Wahlrecht besteht auch in einem Drittstaat. Ferner in 4 Verträgen Argentiniens (Nr. 124, 127, 129, 191: im letzten Fall in Drittstaaten Aufschub) und einem Vertrag Portugals (Nr. 108). Indirekt gilt auch Nr. 131 für

8 Vgl. hierzu die jüngste Monographie von Karin Kamman, Probleme mehrfacher Staatsangehörigkeit, Ffm.1984, 318 S. Auch hierbei handelt es sich um eine Hamburger Dissertation. Vgl. ferner: Hecker, Erleichterungen für Doppelstaater in Konsularverträgen der USA mit kommunistischen Staaten, in: WGO 1986, S. 31-34.

9 A. Karamanoukian, La double nationalité et le service militaire in: RGDIP 1974, S. 459-484; VRU 1971, S. 75-79 (betr. US-Verträge). 
Doppelstaater, obwohl es sich nur um ein allgemeines Wehrpflichtabkommen handelt, insb. für Falkländer.

Gewisse Einschränkungen des allgm. Prinzips der Wahlfreiheit werden getroffen: Sie gilt nur in beiden Weltkriegen (Nr. 26, 27) oder nur für den 2. Weltkrieg (Nr. 18). Sie gilt subsidiär nur bei Wohnsitz in Drittstaaten gemäß drei Verträgen Frankreichs (Nr. 94, 128, 147). Sie gilt nur vor Erreichung des Dienstpflichtalters und Einberufung in 2 Verträgen der Niederlande (Nr. 59, 114). Sie gilt sowohl bei Wohnsitz in Drittstaaten wie vor dem Einberufungsalter von 19 Jahren (Nr. 101, 123, 170, 172; M 14, Art. 6).

\section{Wehrpflicht im Vertragsstaat des Wohnsitzes}

Die Wehrpflicht ist generell dort zu erfüllen, wo im Wehrpflichtalter von 18/19 Jahren der Wohnsitz ist: M 6; M 12, wobei 2 Jahre Aufenthalt als Wohnsitz gilt; die jüngeren Verträge Italiens (Nr. 114, 170, 193, 195) und Frankreichs $(94,101,128)$ sowie erst recht Frankreichs mit Italien (Nr. 172) gingen zu diesem Prinzip über. Lediglich in Nr. 196 gilt der Wohnsitz nur subsidär (s. o.). Schon früher hatte der Vertrag Belgien-Niederlande (Nr. 59) erstmals dies Prinzip eingeführt.

Das Haager StA-Protokoll v. 1930 knüpfte nicht an den Wohnsitz sondern an den gewöhnlichen Aufenthalt an, wenn man mit diesem Staat "tatsächlich am meisten verbunden ist". Obwohl die BRD nicht Partner dieses Protokolls ist, übernahm sie dessen Formulierung in drei Verträgen, die an gewöhnlichen Aufenthalt und Schwerpunkt des Lebens anknüpfen (Nr. 85, 88, 105).

Einige Verträge knüpfen an den häufigsten Aufenthalt im letzten Jahr vor Erreichung des Wehrpflichtalters an (Nr. 123, 147, 152). Wird Wohnsitz in Drittstaaten geregelt, dann gilt dort Wahlfreiheit. In Nr. 193 gilt jedoch als Wohnsitz dann der letzte Aufenthalt in einem der Vertragsstaaten. In einigen Verträgen ist noch eine harte Klausel: Wer

seine Wehrpflicht in einem der Vertragsstaaten erfüllt und dann in den anderen zieht, wird dort erneut wehrpflichtig (Nr. 94, 101, 114, 128, 147).

\section{Besonderheiten}

In den obigen bilateralen Verträgen finden sich noch einige Besonderheiten, die vor allem den StA-Begriff betreffen:

Als Doppelstaater gilt auch, wer einen Anspruch auf die StA hat (Nr. 32, 101). Diplomatenkinder sind im Empfangsstaat wehrpflichtfrei (Nr. 32). Auch wenn die andere StA später verloren wird, bleibt die Befreiung bestehen (Nr. 170). Auch wer ohne doppelte StA seine Wehrpflicht im anderen Staat erfüllt, wird befreit; ebenso wer als Doppelstaater vor Inkrafttreten dort diente (Nr. 195). Ausgeschlossen von dem Vertrag sind alle Eingebürgerten (Nr. 101), bestimmte Eingebürgerte (Nr. 94), auch später Eingebürgerte (Nr. 128), nur nach Volljährigkeit Eingebürgerte (Nr. 123). Solche sind in dem Land dienstpflichtig, in welchem sie sich im Jahr vor der Einbürgerung am meisten aufgehalten haben (Nr. 147, was nach diesem Vertrag auch sonst gilt (s. o.)). 


\section{Förderung der Doppelstaatigkeit}

\section{Allgemeines}

Schon immer gab es außer der Hauptströmung im internationalen und nationalen Recht, welche Doppelstaatigkeit als ein Úbel ansah, das es zu beseitigen und zu verhindern gelte, eine andere Strömung, welche, im diametralen Gegensatz dazu, gerade Doppelstaatigkeit herbeiführte. In Deutschland verlieh man Richtern überstaatlicher Gerichte (Oberappelationsgericht der Freien Städte 1820, OLG Thüringen 1872, OVG Thüringen 1910, preußisch-thüringerscher Vertrag v. 1931) eine mehrfache StA. Ungarische Staatsangehörige in der Waffen-SS erwarben dadurch kraft Vertrages die deutsche StA hinzu (erstmals Vertrag v. 14.4. 1944, Art. 12; hier Nr. 1 v. 14. 2. 1945, Art. 13). In neuerer Zeit nahm der Gesetzgeber der BRD Doppelstaatigkeit in Kauf, weil Gleichberechtigung der Geschlechter und Familieneinheit bei Adoption demgegenüber den Vorrang hätten. Hierher gehört auch die h. M., wonach von der DDR Eingebürgerte außer der DDR-Staatsbürgerschaft auch die gesamtdeutsche StA erwerben.

Im internationalen Bereich hat es bisher, soweit bekannt, nur einen Versuch gegeben, durch einen multilateralen Vertrag Doppelstaatigkeit einzuführen, und zwar 1965 (M 21). "In Anbetracht dessen, daß das Prinzip der Doppelstaatigkeit der beste Weg ist, die afrikanische Einheit zu erreichen" (so die Präambel), wollten die 4 westafrikanischen Vertragspartner ihre Integration dadurch fördern. Dieser Vertrag trat jedoch nie in Kraft.

\section{Motive für Spaniens Doppelstaatenverträge ${ }^{10}$}

Spanien hat bisher mit 12 der 17 spanischsprachigen Republiken Lateinamerikas (außer Mexico, Panama, Salvador, Uruguay, Venezuela) Verträge zwecks Erleichterung des Entstehens von Doppelstaatigkeit geschlossen. Als Motiv für diese Verträge geben ihre Präambeln an:

1. die Gemeinsamkeit, die durch Tradition, Kultur und Sprache (Honduras außerdem: Blut) bestehen (so die ersten 9 Verträge: Nr. 91, 99, 100, 117-119, 132, 133, 140). Die drei jüngeren Verträge schwächen dies ab zu: Tribut an historische Abstammung

10 Hierzu: I. Aznar Sanchez, La doble nacionalidad, Madrid 1977, S. 30-75; A. Boggiano, La doble nacionalidad de derecho int. priv. (con especial referencia al derecho convencional hispanoamericano) in: Revista de derecho intern. 1969, Nr. 35/6, S. 5-65. F. Prieto-Castro y Roumier, La nacionalidad multiple, Madrid 1962, S. 147-165; A. Barbero Garcia, Los convenios hispano-americanos de doble nacionalidad y el registro civil, in: Boletin de Información 1971, Nr. 896, S. 3-12; Nr. 897, S. 3-16; G. B. Taboado, La ley argentina de nacionalidad 21795 y los convenios internacionales con España y Italia, in: El Derecho. Legisl. Argentina 1980, S. $1161 \mathrm{ff}$.: A. Marin Lopez, La doble nacionalidad en los tratados suscritos por España con las repúblicas americanas, in: Anuario de derecho intern. 1982, S. 219-232; J. E. Greño Velasco, La denominada formula argentina en el convenio de nacionalidad con España, in: Jurisprudencia Argentina 1974, Ser. cont. S. 657-673 und La 'clausula constitucionalı en el convenio de nacionalidad entre la República Argentina y España, in: Anuario hispano-luso- americano de derecho int. 1973, S. 207-240. Ferner Clunet 1973, S. 747. 
(Nr. 145, 149, 189) und gemeinsames Substrat (Nr. 145, 149) bzw. gemeinsame Erbmasse und iberoamerikanischer Kulturraum (Nr. 189).

2. Die Angehörigen beider Staaten fühlten sich im jeweils anderen nicht wie Ausländer (Nr. 91, 99, 103, 132, 145) bzw. wie in der Heimat (Nr. 117, 140).

3. Der spanische Código Civil (Art. 23) und die lateinamerikanischen Verfassungen stimmten darin überein, daß sie zu Verträgen ermächtigten, wonach die Angehörigen eines Staates die StA des anderen hinzuerwerben können (Nr. 91, 99, 117, 118, 140; Nr. 132 statt Verfassung ein Dekret von 1963). Nr. 189 spricht von den Verfassungsnormen beider Staaten, wobei Art. 11 III der spanischen Verfassung von 1978 gemeint ist, der solche Verträge mit iberoamerikanischen Staaten und solchen, bei denen "una particular vinculación" mit Spanien bestehe, zuläßt. Ob letzteres wegen der großen Zahl spanischer Gastarbeiter in der BRD für diese zutrifft, verdiente nähere Betrachtung. Art. 22 und 23 C.c. nennen nur Andorra, Philippinen, Äquatorialguinea und Portugal.

4. Durch die Doppelstaaterverträge würden die Bestrebungen gefördert, eine neue iberoamerikanische Rechtsordnung zu schaffen (Nr. 140).

5. Es wird die Úbereinstimmung mit Art. 15 MRE betont, weil die Doppelstaaterabkommen die Gefahr der Staatenlosigkeit reduzierten (Nr. 189).

6. Bedenken gegen Doppelstaatigkeit bestünden nicht, weil im Vertrag stets nur eine der beiden Staatsangehörigkeiten effektiv würde (Nr. 117).

\section{3) Inhalt jener Verträge}

Die Abkommen gelten nur für Gebürtige: nur für nacidos im jeweiligen Inland (Nr. 91), für alle "por nacimiento" (Nr. 118), bzw. "de origen" $(100,133,149,189)$. Oder es werden umgekehrt ausdrücklich Personen ausgeschlossen, die vor Inkrafttreten eine der beiden StA'en durch Einbürgerung erwarben (Nr. 92, 117, 119, 132, 140). Nr. 145 enthält keine Einschränkung, gilt also für jede Art von StA-Erwerb. Im jüngsten Vertrag (Nr. 189) wird außerdem zweijähriger Wohnsitz im anderen Staat gefordert: Wer unter das Abkommen fällt, erhält bei solchem Domizil einen Einbürgerungsanspruch neben der bisherigen StA; die Option für die 2. StA wird registriert und dem anderen Staat mitgeteilt.

Effektiv ist allein die StA des Domizils, d. h. zunächst die neue StA. Bei Rückkehr in den anderen Staat mit Domizilwechsel wechselt automatisch die effektive StA; beide Wechsel sind zu registrieren. Bei Domizilnahme in dritten Staaten gilt als effektiv allein die StA, die vor dem Domizilwechsel effektiv war: In Nr. 100 u. 133 ist dieser Fall ungeregelt. Effektiv bedeutet: nur der Domizilstaat ist zuständig für Pässe, diplomatischen Schutz, Wehrdienst und Rechtsausübung. Letztere ist bei Verstoß gegen den Ordre public jedoch unwirksam: In Nr. 118 und 189 fehlt diese Klausel; in Nr. 149 ist sie nur unwirksam, wenn sie ausdrücklich Verfassungsnormen widerspricht (Art. 8 I).

Wiedererwerb durch Option gibt es für alle diejenigen, die vor Inkrafttreten des jeweiligen Vertrages ihre StA wegen Erwerb der anderen verloren hatten. 9 Verträge erkennen 
allerdings als Verlustgrund nur Verzicht an; die beiden letzten Verträge (Nr. 149 u. 189) lassen jeden Verlustgrund gelten; Nr. 118 schließt nur Verlust wegen Erwerb durch Ehe aus und gilt nur für Gebürtige.

Sondervorschriften bestehen noch in 4 Verträgen:

Nr. 100, Art. 6: Wer wegen ius soli Doppelstaater geworden war, für den gilt als effektiv allein diese StA.

Nr. 118, Art. 8-9: Staatsangehörige eines Staates und deren Familienmitglieder können mit dem $\mathrm{Pa} ß$ des letzten Domizils visumfrei in den anderen Staat reisen.

Nr. 140, Art. 8: Wenn jemand durch die Rechte beider Staaten seine 2 StA'en erhielt, gilt der Vertrag ebenfalls.

Nr. 149, Art. 8 II: Unter besonderen Umständen kann der Vertrag suspendiert werden, ohne daß dadurch die Rechtslage der Betroffenen geändert wird.

\section{Beseitigung bestehender Doppelstaatigkeit}

\section{Allgemeines}

Die Beseitigung einer bestehenden Doppelstaatigkeit erfolgt in erster Linie durch freie Wahl (Option) für eine der beiden StA'en und in zweiter Linie durch automatische Zwangszuweisung.

Abgesehen von den unten ausführlich zu behandelnden Verträgen der Ostblockstaaten unter sich gibt es nur 4 Verträge dieser Art, auch dabei 2 mit einem kommunistischen Staat.

M 20 ist der einzige multilaterale Vertrag und der ist noch nicht in Kraft. Art. 8 sagt: Wird das Optionsrecht nicht ausgeübt, so gilt generell allein die letzterworbene StA; bei gleichzeitigem Erwerb gilt der Wohnsitz.

Nr. 22: Doppelstaater, auch wenn sie die britische StA durch Option erworben hatten, können auf die burmesische StA verzichten.

Nr. 73: Allein die in Tibet wohnenden Doppelstaater können ab 18 Jahren für China optieren, womit automatisch die StA Nepals erlischt.

Nr. 65: Innerhalb von 2 Jahren können Volljährige (und verheiratete Minderjährige) für die eine StA optieren, indem sie auf die andere verzichten. Nach ungenütztem Ablauf der Frist gilt allein die StA des Vaters; nur wo keine Rechtsbeziehung zum Vater besteht oder dessen StA unbekannt ist, gilt die der Mutter (Art. 5). Minderjährige folgen der elterlichen oder väterlichen StA-Option, können aber 1 Jahr ab Volljährigkeit re-optieren (Art. 6). Bei Rückerwerb der anderen StA geht, falls man im Ausland wohnt, die neue StA wieder verloren (Art. 7). Im Inland geborene Kinder erwerben die StA, die beide Eltern bzw. der Vater haben (Art. 8). Vor dem Alter von 5 Jahren Adoptierte wechseln dadurch automatisch die StA (Art. 9). 


\section{Die Ostblock-Verträge ${ }^{11}$}

Abgesehen von sechs Nachtrags-Verträgen der SU, die vor allem die Verhinderung künftiger Doppelstaatigkeit betreffen (Nr. 125, 136, 141, 173c, 184, 191a) und im nächsten Kapitel zu behandeln sind, gibt es 28 Doppelstaaterverträge des Ostblocks, davon nur sieben mit außereuropäischen kommunistischen Staaten, nämlich sechs mit der Mongolei (Nr. 95, 173b, 181, 183, 198, 199) und einen mit Nord-Korea (Nr. 87). Die Sowjetunion begann die Serie dieser Verträge 1956 ausgerechnet mit Jugoslawien, und hat insgesamt 16 Verträge geschlossen. Als nächster Staat folgt die DDR mit 7 (einen davon mit der SU). Die Verträge haben trotz ihrer übereinstimmenden Grundtendenz im einzelnen erhebliche Unterschiede und sind keineswegs uniform. Die Verträge trennen formelle und materielle Vorschriften nicht klar genug und unterscheiden erst in späteren Jahren Beseitigung bestehender und Verhinderung künftiger Doppelstaatigkeit.

\section{a) Volljährige}

Bei Inkrafttreten des jeweiligen Vertrages volljährige d. h. über 18 Jahre alte Doppelstaater kraft Geburt können innerhalb eines Jahres danach eine der beiden StA'en wählen (so alle Verträge). Den Volljährigen gleichgestellt sind verheiratete Minderjährige (Nr. 69, 81-84, 86, 87, 90, 111, 137, 159, 169, 173b, 198, 199) bzw. den Volljährigen gleichgestellte Minderjährige (Nr. 156, 181). Der erste aller Verträge (Nr. 69) erklärte: Wer ohne Genehmigung zur Aufgabe seiner bisherigen StA die StA des zweiten Staates hinzuerworben habe, aber noch auf dem Gebiet des ersten Staates wohne, gelte allein als dessen StA'er.

Wird innerhalb der erwähnten Frist keine Erklärung abgegeben, so bleibt nur die StA des Wohnsitzes bei Fristablauf bestehen, die andere erlischt automatisch (so alle Verträge). Die meisten neueren Verträge sagen noch, daß bei Wohnsitz in einem 3. Staat die StA des Vertragsstaates gilt, in dem vorher der letzte Wohnsitz bestand (Nr. 84, 86, 111 , 136, 137, 148, 151, 156, 159, 164, 169, 173b, 175, 181, 183, 198, 199). Bestand ein solcher nicht, so gilt allein die später erworbene StA (Nr. 137, 169, 199). Bei gleichzeitigem Erwerb gilt dabei die StA der Mutter $(169,199)$. In drei Zweitverträgen (Nr. 136, Art. 5 II; Nr. 141, Art. 8; Nr. 184, Art. 2) wird denjenigen Doppelstaatern die ihre zweite StA nicht durch Geburt sondern vor Inkrafttreten anderweitig, insb. durch Einbürgerung, erworben hatten, kein Wahlrecht gegeben, es gilt nur die StA des Wohnsitzes bzw.

11 I. Sipkov, Settlement of dual nationality in European Communist Countries, in: AJIL 1962, S. 1010-1019; L. Gelberg, Problems of dual nationality in the light of legislation and treaties of the socialist countries, in: Polish Yearbook of int. Law 1966/7, S. 86-110. Ferner Geilke, in SGS Bd. 25, S. 194-199 mit Hinweis auf ungenügende Darstellungen v. Aleksandrikov und Vilkov. Uber die DDR-Verträge: H. v. Mangoldt, Deutsche Staatsangehörigkeitsrecht, 22, S. 1-7. Für die Ubersetzung der Verträge, die in keiner westlichen Sprache vorlagen, habe ich den Mitarbeiter der Ostrechtsabteilung der Universität Hamburg zu danken, die mir freundlicherweise jede Hilfe angedeihen ließen. Eine ausführliche Darstellung der Doppelstaatverträge des Ostblocks ist vorgesehen für WGO Heft 5/1986. Der Text Nr. 19la konnte in vorliegenden Arbeit noch nicht berücksichtigt werden. 
bei Wohnsitz in einem 3. Staat die später erworbene StA (Nr. 136 und 141), oder sofort nur die später erworbene StA (Nr. 184).

\section{b) Minderjährige im Grundgesetz}

Hier wird differenziert, ob die Eltern verschiedene Vertragsstaatsangehörigkeiten haben oder die gleiche. In beiden Fällen gibt es eine starre und eine liberale Lösung.

Erster Fall (gleiche StA):

In allen Verträgen der ersten 10 Jahre (1956-1965) findet sich die rigorose Vorschrift, daß falls die Eltern gemäß dem jeweiligen Vertrag die gleiche StA haben (oder wenn nur noch ein Elternteil lebt: Nr. 111) diese sich automatisch auf die Kinder erstreckt. Ebenso wieder neuerdings (Nr. 164, 169, 175, 184, 187, 188, 198, 199). In anderen der neueren Verträge - Nr. 173b hat ein kombiniertes System - erhalten die Eltern aber innerhalb von einem Jahr ein Wahlrecht (Nr. 148, 151, 156, 159, 164, 181, 183), wobei betont wird, daß die Kinder vor Inkrafttreten des jeweiligen Vertrages geboren sein müssen. Jedoch müssen die Kinder über 14 Jahren (Nr. 159: 16) zustimmen. Nr. 181 bestimmt, daß die Einwilligung sich nach dem gewählten Staat richtet, d. h. auch das Alter, ab welchem Einwilligung nötig ist.

Zweiter Fall (verschiedene StA):

Hat von den Eltern der eine Teil die StA des einen, der andere die StA des anderen Vertragsstaates, so bestimmte der erste Vertrag (Nr. 69) starr, daß die StA des Ortes gelte, wo das Kind gemeinsam mit den Eltern oder einem Elternteil oder als Waise lebe; ab 16 könne es für die andere StA re-optieren. Die anderen alten Verträge gaben den Eltern ein Wahlrecht und meistens ab 14 Jahren dem Kind ein Re-optionsrecht (Nr. 82, 83, 84, $86,87,90)$.

Neuere Verträge geben den Eltern ein Wahlrecht, und zwar meist innerhalb eines Jahres ab Inkrafttreten (Nr. 159, 169, 173b, 173c, 175, 183, 187, 188, 199) bzw. innerhalb von 18 Monaten (Nr. 184). Die Wahl der Eltern hat einvernehmlich und schriftlich zu erfolgen. Die Kinder müssen schriftlich zustimmen, wenn sie über 14 sind (so die Regel) bzw. über 15 (Nr. 183) bzw. über 16 (Nr. 159) bzw. entweder 15 oder 16 (Nr. 173b). Eine Reoption gibt es für die Kinder in diesen neueren Verträgen nicht mehr. Bei Kindern, die zwischen 4. 12. 1959 (Ablauf der Einjahresfrist der Wahl nach Nr. 95) und 11. 9. 1975 (Inkrafttreten v. Nr. 173c) geboren sind, gilt das Gleiche, jedoch müssen bei Wahl der sowjetischen StA die Kinder v. 15-18, bei Wahl der mongolischen die Kinder von 14-18 zustimmen.

\section{c) Ersatzlösungen für Minderjährige i. a.}

Läuft die einjährige Wahlpflicht ohne Erklärung oder Einigung der Eltern ab, so behalten die minderjährigen Kinder allein die StA desjenigen Vertragsstaates, auf dessen Gebiet sie bei Fristablauf gemeinsam wohnen (Nr. 81-84, 86, 87, 90, 95, 111, 148, 151, 156, 159, 169, 173b, 173c, 175, 181, 183, 184, 187, 188, 198, 199). Dazu Besonderheiten: 
Erstens: fehlt es an einem gemeinsamen Wohnsitz der Eltern, dann gibt es 3 Varianten:

1) Leben die Eltern in verschiedenen der beiden Vertragsstaaten, so können sie die StA des Kindes durch freie Vereinbarung regeln; mangels einer solchen gilt für das Kind die StA des Staates, dem der faktisch erziehende Elternteil angehört (Nr. 82-84, 86, 87, 90, 95, 111).

2) Leben die Eltern getrennt, so gilt das Gleiche (Nr. 81): Getrenntleben bedeutet hier of fenbar gewollte Trennung; es wird auch nicht Wohnsitz in einem Vertragsstaat gefordert.

3) Haben die Eltern keinen gemeinsamen Wohnsitz, dann gilt für das Kind die StA des Staates, dem der faktisch erziehende Elternteil angehört (Nr. 164) bzw. der Sorgeberechtigte (Nr. 92, 103 u. 199) bzw. wo das Kind lebt (Nr. 169, 198). Wurde das Sorgerecht aber einem Elternteil gerichtlich übertragen, dann gilt dessen StA auch bei gemeinsamem Wohnsitz (Nr. 199).

Zweitens: Lebt das Kind aber in einem 3. Staat, dann gilt:

1) Es gilt primär der letzte Wohnsitz der Eltern in einem Vertragsstaat (Nr. 84, 86, 92, 103, 111, 116, 137, 159, 164, 169, 173b, 175, 181, 183, 187, 198, 199). Dies gilt generell auch, wenn das Kind in einem 3. Staat geboren ist (Nr. 111) oder nur dann (Nr. 184).

2) Besteht kein solcher letzter Wohnsitz, dann gilt entweder die StA der Mutter (Nr. 159, 164, 169, 173b, 175, 181, 183, 199), oder die zuletzt erworbene StA (Nr. 137).

3) Ist einem Elternteil das Sorgerecht zugesprochen, so können sie die StA des Kindes bestimmen; kommt es zu keiner Einigung, so gilt die StA des Sorgeberechtigten (Nr. 169).

\section{d) Ausfälle bei den Eltern}

\section{Erstens: Ausfall eines Elternteils:}

War ein Elternteil schon vor Inkrafttreten des Vertrages verstorben oder sein Aufenthalt unbekannt (Verschollenheit) oder war ihm das Sorgerecht entzogen, dann galt die StA des anderen sorgeberechtigten Elternteils (Nr. 159, 164, 169, 173b, 175, 181, 183, 187, 188, 198, 199; Nr. 116, 137, 148, 151, 173b ohne Verschollenheit).

\section{Zweitens: Ausfall beider Elternteile:}

Waren beide Eltern verstorben, verschollen, oder war ihnen das Sorgerecht entzogen, dann erwarb das Kind die StA seines Wohnsitzes (Nr. 136, 148, 151, 156, 164, 169, 173b, 175, 181, 183, 187, 188, 199; nur bei Vollwaisen: Nr. 81-84; nur bei Vollwaisen u. Verschollenen: Nr. 92, 103, 116).

Besonderheiten:

1) Bei Vollwaisen u. Kindern Verschollener entscheidet der Vormund über die StA (Nr. 137).

2) Bei Kindern über 14 Jahren ist Re-option möglich (Nr. 103).

3) Bei Wohnsitz in einem dritten Staat gilt die StA des letzten Elternwohnsitzes vor Ausreise bzw. ersatzweise die StA der Mutter (Nr. 116, 136, 169, 173b, 183, 199). 
4) Wählen bei einem Kind unter Vormundschaft die Eltern nicht die StA des Staates, in dem sie nicht wohnen, dann gilt der Wohnsitz des Kindes im 1. Jahr. Wohnte das Kind in einem dritten Staat, gilt die StA des Staates vor der Ausreise, ersatzweise die der Mutter (Nr. 116, Art. 4, Abs. 7-8).

\section{Drittens: Unehelichkeit, Scheidung, Getrenntleben:}

Erst in 5 neueren Verträgen ab 1965 (davon allein 4 Verträge der DDR) werden diese Fälle berücksichtigt. Dabei wird außer Scheidung auch Nichtigerklärung der Ehe aufgezählt $(\mathrm{Nr} .137,148,156)$ oder es wird nur erwähnt, daß die Ehe nicht mehr besteht (Nr. 151). Ein Vertrag berücksichtigt nur Unehelichkeit u. erwähnt Scheidung und Getrenntleben gar nicht (Nr. 181). Ein anderer Vertrag läßt das Getrenntleben unberücksichtigt (Nr. 156).

Die Eltern haben hier freie Wahl, welche StA sie für das minderjährige Kind wählen wollen. Uben sie die Wahl nicht aus (oder stimmt das Kind über 14 nicht zu: Nr. 151), dann gilt die StA dessen, dem gerichtlich das Sorgerecht übertragen wurde (Nr. 137) oder wem es obliegt (Nr. 148) oder wer es faktisch ausübt (Nr. 148 bei Getrenntleben; Nr. 151, 156, 181).

\section{Verhinderung künftiger Doppelstaatigkeit}

\section{Grundsätzliches}

Hierbei gibt es 2 ganz verschiedene Fälle: Doppelstaatigkeit wird verhindert, wenn bei Einbürgerung die bisherige StA erlischt. Dieser Fall wird erst ab 1963 in den Doppelstaatenverträgen des Ostblocks behandelt und ist zweckmäßigerweise erst unten im Kapitel über die Einbürgerung näher zu erörtern.

Der andere, hier allein zu behandelnde Fall, betrifft Personen, die nach Inkrafttreten des jeweiligen Vertrages geboren sind und Doppelstaater geworden wären, wenn die Vertragsvorschriften es nicht ex tunc verhindern würden. Dieser Fall wurde erst in dem fünften der 34 Doppelstaatenverträge entdeckt (Nr. 84) Rechtstechnisch gab es nun 3 Regelungsmöglichkeiten:

1. Der Fall wurde nur in einem einzigen Vertragsartikel geregelt und dabei wurden gegebenenfalls andere Vorschriften analog herangezogen (Nr. 84, 86, 92, 111, 116, 137, $148,151,175,183,187,188)$.

2. Für sechs Verträge der SU, die vor ausführlicher Regelung dieses Falles geschlossen waren, wurde nachträglich durch einen weiteren Vertrag dieser Fall neben anderen Ergänzungen des alten Vertrages gesondert geregelt (Nr. 125, 136, 141, 173 c, 184, 191 a), wobei das Protokoll zu Nr. 141 ausdrücklich Nr. 86, Art. 5 aufhob.

3. In den weiteren jüngeren Verträgen wurde der Fall in einem besonderen Kapitel des Vertrages in mehreren Artikeln geregelt (Nr. 103, 156, 159, 164, 169, 181, 198, 199; Nr. 173 b ohne Kapiteleinteilung). 
Im Grundsatz wählen die Eltern eine StA für das Kind, was auf die Geburt zurückwirkt, so daß nie eine Doppelstaatigkeit entstand. Die Frist dafür beträgt nur drei Monate (92, 103, 136, 137, 164, 173 b, 173 c, 184, 187, 199) oder 6 Monate $(116,159,169,175,181)$ oder meist ein Jahr $(84,111,125,141,148,151,156,183,188,198)$.

\section{Ersatzlösungen}

Haben die Eltern die Erklärungsfrist verstreichen lassen, dann greift ein automatischer Zuteilungsschlüssel ein, der entweder auf Geburtsort oder Wohnort abstellt, aber bei Nr. 136, 148, 151, 159, 169 ohne Rückwirkung: Das Kind erhält die StA des Staates, in dem es geboren ist (Nr. 84, 86, 136, 137, 148, 156, 159, 169, 173 b, 175, 181, 187, 198, 199). Das Kind erhält die StA des Wohnsitzes der Eltern entweder bei der Geburt des Kindes (Nr. 103) oder bei Fristablauf (Nr. 92, 111, 116, 125, 151, 164, 173 c, 183, 184, 188).

Wurde das Kind in einem 3. Staat geboren, so gilt der letzte gemeinsame Wohnsitz der Eltern in einem der Vertragsstaaten, wie alle Verträge (außer zwei alten: Nr. 92 u. 103) hervorheben. Fehlt ein solcher Wohnsitz, so gilt die StA der Mutter. Wenn ihr das Sorgerecht entzogen wurde (oder sie gestorben ist: Nr. 169) gilt die StA des Vaters (letzteres erst in Verträgen ab 1963, ohne Nr. 136, 137, 173 c, 183, 198, 199).

\section{Ausfälle bei den Eltern}

Erst ab 1963 sind diese Fälle berücksichtigt.

\section{Tod, Verschollenheit, Sorgerechtsentzug:}

Ist nur ein Elternteil derart ausgefallen (Fall A), dann entscheidet der andere Teil. Sind beide Elternteile davon betroffen (Fall B), dann entscheidet der letzte Wohnsitz der Eltern bei Fristablauf (Nr. 125, 141, 148, 156, 159, 164, 173 b, 173 c, 175, 181, 183, 184, $187,188,198$ ) bzw. die StA des Staates, in dem das Kind geboren ist oder ersatzweise die StA der Mutter (Nr. 199). Nicht genannt ist Sorgerechtsentzug bei A in Nr. 141, 148, 184 und bei B in Nr. 125 und 141. In Nr. 156 ist bei B entscheidend, bei welchem Elternteil das Kind wohnt oder wer das Erziehungsrecht ausübt. Nr. 164 verweist auf den normalen Verteilungsschlüssel, so daß es bei Fehlen eines letzten Wohnsitzes auf die StA der Mutter ankommt.

\section{Unehelichkeit, Scheidung, Getrenntleben:}

Es gilt die StA des Erziehungsberechtigten, und zwar in allen 3 Fällen (Nr. 148, 151, 173 c, 183, 198); nicht bei Unehelichen (Nr. 141, 156), nur bei Getrenntleben (Nr. 125, 164), notfalls bei Getrenntleben die StA des Wohnsitzes des Kindes (Nr. 198). Sonderfall Nr. 199: Hat die Mutter eines unehelichen Kindes die StA des einen Vertragsstaates und ist die Vaterschaft für den anderen Staat festgestellt, dann können die Eltern innerhalb von 3 Monaten ab dieser Feststellung die StA wählen, wobei Kinder über 14 Jahren 
zustimmen müssen. Wählen sie nicht, so erhält das Kind die StA der Mutter. Bei Getrenntleben verheirateter Eltern gilt das gleiche, nur entscheidet hier bei Nichtwahl die StA des Sorgeberechtigten (Art. 8-9).

\section{Sonderfälle}

6 Sonderfälle sind geregelt in: Nr. 92 (Art. 9, 1), Nr. 136 (Art. 3), Nr. 164 (Art. 8), Nr. 173 c (Art. 1, 3 a), Nr. 188 (Art. 4, 3), Nr. 188 (Art. 7).

\section{B. Staatsangehörigkeit und Gebietswechsel}

\section{Automatischer und absoluter Staatsangehörigkeitswechsel}

M 5 (Art. 10) verpflichtet die Staaten, bei Gebietswechsel vertraglich vorzusehen, daß niemand staatenlos wird. Kommt es nicht zu einem solchen Vertrag, so besteht eine Pflicht, denjenigen die StA zu verleihen, die sonst staatenlos würden.

In bilateralen Verträgen findet sich dies Prinzip nur noch in 4 Dekolonisierungsfällen:

Die "Einwohner" der Kuria-Muria-Inseln verloren ihre britische und erwarben die omanische StA mit dem Gebietswechsel (Nr. 144). Die "Bewohner" der früher japanischen Insel Formosa (Taiwan) verloren ihre japanische StA und galten nur noch als Chinesen seit der Abtretung (Nr. 48).

Wer gemäß Art. 8 des Friedensvertrages Frankreichs mit Thailand vom 9. 5. 1941 die StA im abgetretenen indochinesischen Gebiet gewechselt hatte, erhielt 1946 automatisch wieder die französische StA. Die Bewohner, die dort die thailändische StA hatten oder gemäß Gesetz erworben hatten, behielten aber außerdem auch diese StA und konnten frei Indochina verlassen (Nr. 14). Hier liegt also zwar 1941 automatischer Wechsel vor, 1946 aber nur automatischer Wiedererwerb ohne Wechsel. ${ }^{12}$

Im gescheiterten Mikronesien-Compact (Nr. 179) war vorgesehen: ${ }^{13}$ Citizens des Treuhandgebietes, die keine Schritte für Erwerb oder Beibehalt einer dritten StA unternommen hatten oder Personen, die später durch Gesetz Citizens von Mikronesien wurden, gelten automatisch als US-nationals. Citizens des Treuhandgebietes, die außerdem eine StA eines dritten Staates besitzen, verlieren die US-nationality, falls sie nicht auf die Dritt-StA verzichten.

\section{Automatischer StA-Wechsel mit Beibehalts-Option}

Dies gibt es mit einem Abwanderungsrecht der Optanten oder mit einer Abwanderungspflicht oder ohne solche Regelung.

12 Dazu SGS Bd. 34, S. 148.

13 Dazu SGS Bd. 37, S. $168 \mathrm{f}$. 


\section{Mit Abwanderungsrecht}

Diese Regelung findet sich nur in Asien, und zwar in je 2 Verträgen Indiens (Nr. 42, 70) $)^{14}$ und Chinas $(110,121){ }^{15}$

Wer bei der Abtretung Chandernagores dort wohnte, wechselte automatisch die StA, konnte aber rückwirkend 6 Monate lang für Beibehaltung der französ. StA optieren und nach Frankreich abwandern (Nr. 42).

Wer bei der Abtretung des übrigen Franz.-Indien dort als französ. StA geboren war und dort oder in Indien wohnte, wechselte ebenfalls wie oben; wohnte er dagegen anderswo, so behielt er die französ. StA, konnte dann aber für Indien optieren (Nr. 70).

Nr. 121 sah automatischen StA-Wechsel der Einwohner des abgetretenen Gebietes vor, mit Optionsrecht für Beibehalt innerhalb eines Jahres mit freiem Abwanderungsrecht.

Nr. 110 sah das gleiche vor, ohne aber eine Optionsfrist festzulegen: es wurde statt freier Abwanderung von Möglichkeit der Umsiedlung gesprochen, was unfreiwillige Abschiebung nicht auszuschließen scheint.

\section{Mit Abwanderungspflicht}

Dergleichen findet sich ausschließlich bei Italien in der friedensvertraglichen Regelung. ${ }^{16}$ M 23 hatte für alle abgetretenen Gebiete folgenden Grundsatz: Wer 1940 im abgetretenen Gebiet wohnte und die später geborenen Kinder wechselten automatisch die StA, hatten aber ein Optionsrecht. Bei diesen Optanten hatte der andere Staat das Recht zu verlangen, daß sie in 1 Jahr nach Italien abwanderten (Art. 19; ebenso für Triest). Jugoslawisch Sprechende in Italien konnten für Jugoslawien optieren und mußten dann abwandern (Art. 20). Speziell im Verhältnis zu Jugoslawien gab es noch 4 bilaterale Verträge: (Nr. 41, 47, 64, 135): Dadurch wurden insb. die Fristen in Art. 19 und 20 von M 23 verlängert, das Verfahren der Anerkennung von Optionen erleichtert, die Wiederaufnahme abgelehnter Optionen ermöglicht, StA-fragen der Nicht-Optierer geregelt usw. Speziell für Triest wurde in Nr. 174 vorgesehen: ${ }^{17}$ Am 10. 6. 1940 (Kriegseintritt) im Triester-Gebiet wohnende italienische StA'e und ihre seitdem geborenen Kinder unterliegen der StA des Landes, in welchem sie am 3. 4. 1977 wohnen, womit ev. ein automat. StA-Wechsel eintritt. Jeweils andere Volkszugehörige können für Abwanderung optieren und wechseln dann mit Grenzübertritt wiederum automatisch die StA, wobei Italien anerkennt, daß seine StA nie verloren wurde.

14 Dazu SGS Bd. 26, S. 44 f.; dazu RCDIP 1973, S. 371 u. 1975, S. 828.

15 Dazu SGS Bd. 26, S. 61.

16 Dazu SGS Bd. 27, S. 97-114.

17 S. A. Sinagra, Problemi di cittadinanza e di indemnizzi nel trattato italo-jugoslavo di Osimo di 1975, in: RDI 1977, S. 485-506. 


\section{Ohne Abwanderungsregelung}

Nr. 146: Dieser Vertrag spricht mit keinem Wort von Automatik, setzt sie aber als selbstverständlich voraus, denn Art. 3 gibt bestimmten Spaniern, die in Ifni geboren sind, 3 Monate lang ein Optionsrecht, die spanische StA beizubehalten. Diese gelte nach Art. 1 des Protokolls dann als Verzicht auf die (automatisch erworbene) marokkanische StA. Keinen automatischen StA-Wechsel gab es bei denjenigen Spaniern, die ihre StA nach den Weisen des C.C. erworben hatten (also insb. Nicht-Eingeborene): sie blieben Spanier. Für beide Gruppen von Spaniern gab es weder ein Recht noch eine Pflicht zur Abwanderung.

\section{Reine Option ohne Automatik}

\section{Mit Abwanderungsrecht}

Nr. 33: Italienischsprachige (auch ohne ital. StA), die am 10. 6. 1940 auf dem Dodekanes wohnten, diesen aber bis 31. 8. 1949 verließen, gelten so, als hätten sie für Italien optiert, können aber 2 Monate lang für Griechenland optieren und dahin abwandern. ${ }^{18}$ Nr. 74: Deutsche StA'e im abgetretenen Gebiet (60 Personen) behalten ihre StA, können aber 2 Jahre lang für Belgien optieren (damit verloren sie die deutsche StA nach $\S 25$ RuStAG). Wer nicht optierte, konnte dort wohnen bleiben oder nach Deutschland abwandern. ${ }^{19}$

\section{Ohne Abwanderungsregelung}

Nr. 15: Im Libanon geborene türkische StA'e, die in einem dritten Staat wohnen, könnten für den Libanon optieren, wenn sie rassisch der Bevölkerungsmehrheit ihres Wohnstaates zugehörigen (Protokoll v. 30. 3. 1940).

Nr. 76: Deutsche StA'e, die am 1. 1.1957 die saarländische StA besaßen, konnten bis 1. 1. 1959 die deutsche StA aufgeben, falls sie dadurch nicht staatenlos würden, und konnten im Saarland wohnen bleiben (trotz $§ 24$ RuStAG). Wer nur die saarländische aber nicht die deutsche StA besaß, konnte für diese optieren. ${ }^{20}$

Nr. 106: Die Deutschen im Abtretungsgebiet (23 Personen) behielten die deutsche StA, konnten aber 2 Jahre lang für die niederländische optieren, wenn sie über 18 Jahre waren und noch in den Niederlanden wohnten. ${ }^{21}$

18 SGS 25, S. $105 \mathrm{f}$.

19 Makarov, Deutsches Staatsangehörigkeitsrecht, 2. Aufl., 1971, S. 579-581.

20 A.a.O., S. 582-586.

21 A.a.O., S. 590-593. 


\section{Gemischte Systeme der Aufteilung von Staatsangehörigen}

Abgesehen von einem ungarisch-rumänischen StA-Bereinigungsvertrag (Nr. 28) geht es hier um die Aufteilung der StA'en bei Unabhängigwerden französischer (Nr. 66, 67), niederländischer (Nr. 34, 176) und britischer (Nr. 109) Kolonialgebiete, wobei Automatik und Option kombiniert werden.

Nr. 28: Wer am 30.8. 1940 und am 10.2. 1949 in einem der Vertragsstaaten in den heutigen Grenzen wohnte, gleich welcher StA, erhält dort einen Anspruch, sich als dortiger StA'er registrieren zu lassen. Unterläßt er dies, so behält er die StA, die er am 30. 8. 1940 hatte. (Art. 1). Wer nach dem 30. 8. 1940 in den anderen Staat zog, ist StA'er des Staates, in dem er am 30. 8. 1940 wohnte, falls er nicht ausgebürgert wurde oder deutschen/ital. Wehrdienst leistete (Art. 2) Art. 1-2 gelten nicht für die, welche sich mit einem gültigen $\mathrm{Pa}$ im anderen Vertragsgebiet aufhalten oder nach 4. 4. 1945 ihre StA durch Antrag wechselten. (Art. 3). Wer nicht die StA seines Wohnortes hat, kann für diese optieren, wenn er v. 30. 8. 1940 bis 1. 1. 1948 dort wohnte (Art. 4). Angehörige eines Vertragsstaates, die im anderen wohnen (Art. 1 o. 4) und Reisepapiere erhielten, können abwandern (Art. 7). Entziehung einer StA bewirkt nicht den Wiedererwerb der früheren StA (Art. 11). Der Vertrag gilt nicht für frühere StA'e eines Vertragsstaates, die die StA eines 3. Staates erworben haben. (Art. 12).

Nr. 66: Tunesien kann seine StA außer in den Fällen der Art. 8-14 frei regeln (Art. 7). Es gibt keine kollektive Verleihung seiner StA an französische StA'e; und es beansprucht keine in Frankreich eingebürgerten oder im Personenstandsregister als Franzosen eingetragene Tunesier; Frankreich entläßt in Tunesien Eingebürgerte aus seiner StA (Art. 8). In Frankreich geborene Kinder eines tunesischen Vaters und einer französischen Mutter können, bei Wohnsitz in Tunesien, auf die französ. StA ein Jahr vor der Volljährigkeit verzichten (Art. 9). Sind sie in Tunesien geboren, so können sie unabhängig vom Wohnsitz auf die tunesische StA verzichten (Art. 10). In Tunesien wohnende Ausländer können die französ. oder tunesische StA durch Einzeleinbürgerung erwerben (Art. 11). Für eine Úbergangszeit von 15 Jahren gilt: In Tunesien von einem ausländischen Vater Geborene behalten dessen StA, falls sie nicht durch Abstammung mütterlicherseits Franzosen sind. Haben sie eine tunesische Mutter, können sie 1 Jahr vor Volljährigkeit für deren StA optieren. In Tunesien von Ausländern Geborene, deren einer Elternteil ebenfalls dort geboren ist, sind Franzosen, können aber die französ. StA ausschlagen und für die tunesische optieren (Art. 12).

Nr. 67: Die französische StA behalten diejenigen, die beider Angliederung Cochinchinas und der Konzessionen an Vietnam dort wohnten, wenn sie nichtvietnamesischer Abstammung waren (Art. 2). Stammten sie aus jenen beiden Gebieten, so erwarben sie die StA v. Vietnam (Art. 3). Für beide Personengruppen gab es aber u. U. jeweils ein Optionsrecht für Frankreich oder Vietnam (Art. 4-5). Für Mischlinge galt: Volljährige wurden Franzosen, wenn ein Eltern- oder Großelternteil es war; Minderjährige folgten dem Vater, stets aber gab es Optionen für die andere StA (Art. 6-10). Der Vertrag 
schließt die Vorschriften der beiderseitigen StA-Gesetze über Erwerb wegen Geburtsort und Wohnsitz aus (Art. 20). ${ }^{22}$

Nr. 34: ${ }^{23}$ Volljährige niederländische StA'e, die in Indonesien wohnten, behielten ihre StA (Art. 3). Eingeborene Kolonialuntertanen erwarben automatisch die indonesische StA, wenn sie dort oder im Ausland geboren waren; waren sie in den Niederlanden, Surinam und Curacao geboren, und wohnten sie dort, so erwarben sie die niederl. StA (Art. 4). Nicht zur indonesischen Rasse gehörende Kolonialuntertanen erwarben je nach Geburtsort und Wohnsitz entweder die indonesische oder die niederl. StA (Art. 5-7). Bestimmte der bisher Genannten konnten innerhalb von 2 Jahren für die andere StA optieren: sie mußten dafür auf die bisherige StA ausdrücklich verzichten. Nach Auffassung der Niederlande wirkte die Option auf die Unabhängigkeit zurück, nach Meinung Indonesiens erfolgte dagegen ev. ein zweimaliger StA-Wechsel. Bei dieser Regelung wurde Neuguinea ausgeklammert.

Nr. 176:24 Grundsätzlich verlor derjenige, der eine der beiden StA'en erwarb, damit automatisch die andere (Art. 2). Die StA von Surinam erwarben die volljährigen dort geborenen Niederländer, die dort am Stichtag wohnten (Art. 3). Die außerhalb geborenen erwarben sie nur, wenn der Vater (bei Unehelichen die Mutter) dort geboren waren und wenn sie die StA durch Einbürgerung, Ehe oder durch Vertrag Nr. 34 erworben hatten (Art. 4). Bestimmte Niederländer konnten außerdem bis 1986 die StA von Surinam durch Option erwerben (Art. 5); in anderen Fällen gab es in 1-3 Jahren Optionen für beide Staaten (Art. 8-10).

Nr. 109: Briten, die ihre StA durch Cyprus Orders 1914-1943 erworben hatten oder ab 5. 11. 1914 auf Zypern geboren waren oder als Mann von diesen abstammten, erwarben die StA von Zypern (Sec. 2). Sie verloren damit die britische StA, außer in 11 Sonderfällen (Sec. 3), konnten sie aber wiedererwerben (Sec. 7). Wer die StA Zyperns mangels Wohnsitz nicht nach Sec. 2 erwarb, erhielt in bestimmten Fällen einen Optionsanspruch auf sie, womit die brit. StA erlosch (Sec. 4 (1) und (14)). Wer am 5. 11. 1914 die türkische StA besaß und auf Zypern wohnte (oder von solchen abstammte), ohne die brit. StA zu besitzen, erhielt ebenfalls einen Optionsanspruch (Sec. 4 (2)). Bestimmte Briten, die innerhalb von 5 Jahren nach Zypern zogen, konnten gleichfalls optieren (Sec. 5). Zypern sollte Doppelstaatern einen Anspruch auf Verzicht auf die StA Zypern geben (Sec. 8), was durch Sec. 7 des StAG v. 1967 auch geschah. 


\section{Einbürgerung und sonstiger Antragserwerb}

\section{Allgemeines}

Multilaterale Verträge sehen ein Wohlwollensgebot bei der Einbürgerung von Flüchtlingen (M 1) und Staatenlosen (M 2) vor, bzw. ein erleichtertes Einbürgerungsverfahren für Angehörige nordischer Staaten (M 10 u. 17).

Bei den bilateralen Verträgen findet sich in 7 Abkommen der USA über Wehrdienstbefreiung (Nr. 3-6, 13, 25, 36) die Klausel, auf solche Befreiung könne sich nicht mehr berufen, wer bereits in den USA einen Einbürgerungsantrag gestellt habe. Umgekehrt heil3t es zusätzlich in 2 Verträgen (Nr. 13 u. 36), wer sich auf die Wehrdienstbefreiung berufe, dem könne die Einbürgerung verweigert werden.

In drei Auslieferungsverträgen der Bundesrepublik (Nr. 44, 153 und 183 a) ist eine Einbürgerung des Auszuliefernden untersagt, es sei denn, er besäße einen Einbürgerungsanspruch (so nur Nr. 153). ${ }^{24 a}$

Gewisse Einschränkungen der Einbürgerung gibt es in drei Verträgen Frankreichs: Jeder Staat kann nach Einbürgerungsankündigung 6 Monate lang widersprechen (Nr. 67, Art. 19). Frankreich wird bei Widerspruch Monacos nicht einbürgern und dieses wird bei Einbürgerungen französische Sicherheitsinteressen berücksichtigen (Nr. 21); das Saarland konnte eine Einbürgerung wegen besonderer Verdienste nur vornehmen, wenn Frankreich zustimmte (Nr. 38).

Einbürgerungsansprüche gibt es in drei Verträgen: Wer Einbürgerungsbedingungen erfüllt und zwei Jahre ununterbrochen im anderen Staat wohnt, hat einen Anspruch auf Einbürgerung bzw. Wiedererwerb (Nr. 23, Art. 25). Wer mit einer Staatsangehörigen des anderen Vertragsstaates verheiratet ist und dort wohnt, hat bei Loyalität einen Einbürgerungsanspruch im Staat der Ehefrau (Nr. 23, Art. 26). Priester im Rahmen eines Konkordats haben einen solchen Anspruch (Nr. 142), ebenso in Neuseeland wohnende oder nach Vertragsschluß legal einwandernde Westsamoaner (Nr. 197). ${ }^{25}$

\section{Austausch von Einbürgerungsmitteilungen}

Es bestehen drei multilaterale Verträge (M 20, Art. 9; M 15; M 14 Protokoll, Art. 1) vier bilaterale Abkommen europäischer Staaten (Nr. 16, 109, 130, 171), 18 Vereinbarungen der Bundesrepublik (s. u.) und Klauseln in 9 Verträgen mit Lateinamerika (Nr. 91, 99, 117-119, 132, 133, 140, 145).

24a Dazu OLG Karlsruhe v. 5. 3. 1986 (NJW 1986, S. 3035).

25 S. NZLJ 1982, S. 273, 353-355; Otago LR 1983, S. 367-396. 


\section{1) Wie oft?}

Selten nur jährlich (Nr. 112), öfter halbjährlich (Nr. 16, 52, 93, 104, 122), meist vierteljährlich (Nr. 43, 45, 46, 49, 53, 78, 97). Einige Verträge sehen sogar auf diplomatischem Wege Mitteilungen für jeden Einzelfall vor (M 15, M 20, Nr. 50, 75, 130, 171, 180), meist sofort; bei den beiden multilateralen Verträgen nach einem Vierteljahr (M 20) bzw. halben Jahr (M 15); nach 60 Tagen bei 8 der spanisch-südamerikanischen Verträge (außer Nr. 118 - ohne Frist).

\section{2) Alte Pässe usw. einziehen?26}

Dies wird in den meisten Fällen ausdrücklich vorgeschrieben: die ungültigen StA-Papiere sind dem Staat der früheren StA zu übersenden. Sonderfälle: Es sind nur Fotokopien davon zu übersenden (Nr. 78). Es wird überhaupt nicht direkt über Einbürgerungen berichtet, sondern es werden nur die dadurch ungültig gewordenen Pässe und StA-Ausweise ausgetauscht (Nr. 51). Es werden überhaupt keine Pässe usw. ausgetauscht, sondern sie werden zu den Akten des einbürgernden Staates genommen (Nr. 57).

\section{3) Ausdehnung auf andere StA-Fälle?}

Am umfassendsten ist die Mitteilungspflicht bei 8 der spanisch-lateinamerikanischen Verträge: dort ist jeder Fall von Erwerb oder Verlust der StA und von Domizilwechsel mitzuteilen. Beim Vertrag mit Honduras (Nr. 140) ist Verlust nicht mitzuteilen. Jeder Antragserwerb ist mitzuteilen (Nr. 171), ${ }^{27}$ Option u. Wiedererwerb (M 15, Nr. 130), auch bestimmte Optionsfälle (Nr. 16, 45, 97, 112). Nur auf Verlangen ist über andere Fälle von StA-Erwerb Auskunft zu geben (Nr. 75).

\section{4) Besonderheiten}

Mitzuteilen sind auch Beibehaltungserklärungen bei Einbürgerungen, wobei Doppelstaatigkeit entsteht (Nr. 97). Aufzuführen sind alle Angehörigen, auf die sich eine Einbürgerung erstreckt (Nr. 50 u. 52). Zu melden sind auch Statusdeutsche (Nr. 45, Nr. 46 Erg., 75, 112, 122, 180). Die Niederlande übersenden nur die Nummern des Staatsblads, in welchem alle Einbürgerungen aufgelistet werden, und kreuzen deutsche StA an (Nr. 46). Eine lediglich einseitige Pflicht besteht für Zypern, das Großbritannien Optionen für dessen StA mitteilt (Nr. 109, Annex Art. 4, 15 und Art. 5, 6). 


\section{Einbürgerung nur bei Verlust bisheriger StA}

Diese Vorschrift zur Verhütung von Doppelstaatigkeit findet sich seit Art. 7 der Haager StA-Konvention v. 1930 in weiteren 4 multilateralen Abkommen: so M 5, Art. 7 (2) u. M 14 (automatischer Verlust bei Einbürgerung oder Option; keine Beibehaltung möglich), ferner M 19, Art. 1 II 2 (Erwerb im Wohnstaat läßt Geburts-StA verlieren) und M 20, Art. 6 (Einwilligung der Regierung der bisherigen StA nötig), Art. 7 (Verlust bei Option).

An bilateralen Verträgen finden sich zunächst folgende 7; dabei die Mehrzahl nur einseitig:

Nr. 21: nur Monaco verpflichtet sich, allein einzubürgern, wer frei von anderer StA Nr. 28, Art. 10: Einbürgerung oder Wiedererwerb erst, wenn aus alter StA entlassen Nr. 40: Osterreich verpflichtete sich, mindestens $25 \%$ der dort wohnenden Südtiroler einen Einbürgerungsanspruch zu geben, wodurch deren ev. bestehende italienische StA erlöschen würde (Ziff. 1)

Nr. 63: keine Einbürgerung ohne Entlassung. Diese Klausel hat größte Schwierigkeiten für die Einbürgerung von Iranern in der Bundesrepublik bereitet und es sind die verschiedensten Versuche unternommen worden, sie zu umgehen, da der Iran meist eine Entlassung verweigert. ${ }^{28}$

Nr. 67: Vietnamesen in Frankreich und Franzosen in Vietnam erhielten einen Einbürgerungsanspruch (mit Widerspruchsrecht des Aufenthaltstaates), womit ein Verlust der bisherigen StA eintrat (Art. 19) ${ }^{29}$

Nr. 120: Franzosen in Algerien, die ihr Personalstatut behielten, aber schon algerische Bürgerrechte ausüben konnten, erhielten einen Anspruch, nach der Unabhängigkeit drei Jahre lang durch Antrag der Aufnahme in die Wählerlisten statt dessen allein die algerische StA zu erwerben. ${ }^{30}$

Nr. 146, Protokoll: Bei Eintragung in spanische Register muß vorher auf marokkanische StA verzichtet sein.

Nr. 161: Verfahren der Entlassung aus polnischer StA, um die der USA zu erwerben

Nr. 168: Wer freiwillig die malayische StA erwarb oder erwirbt, verliert automatisch die chinesische. Hier wird also zwar nicht die Einbürgerung von der Entlassung abhängig gemacht, sondern der Verlust von der Einbürgerung, was aber praktisch zu einer Einwilligung zur Entlassung gleichkommt. ${ }^{31}$

Vor allem aber findet sich das Erfordernis des Verlustes der bisherigen StA bei Einbürgerung in vielen Doppelstaatenverträgen des Ostblocks. (Note zu Nr. 92; Nr. 136, 137, $151,156,159,164,169,181,183,184,187,188,198,199)$. In weiteren 5 Verträgen - da-

28 VGH. Stuttgart v. 30. 3. 1981 u. BVerw. v. 10. 7. 1984 (kein Vorrang v. Art. 6 I GG); OVG Münster v. 21.2. 1984 u. VG Wiesbaden v. 29. 8. 1984 (Vorrang v. Art. 6 I GG); Heldmann (IBAR 1984, S. 315), bestreitet Gültigkeit der Wiederanwendung des Vertrages im Jahre 1954.

29 Zu Art. 19 s. Zirkular v. 29. 5. 1965 (J.O. v. 6. 6. 65, S. 4676).

30 S. SGS Bd. 39, S. 9 (Parl. Anfragen 1963-1979).

31 S. SGS Bd. 36, S. 104. 
von 4 der SU - wird Beibehaltung der bisherigen StA nicht ausgeschlossen (Nr. 125, 141, 148, 173 c, 175), also Doppelstaatigkeit ermöglicht.

\section{Optionen ohne Gebietswechsel und Umsiedlung}

Abgesehen von Optionen im Zusammenhang mit Gebietswechsel (s. o.B) und Bevölkerungswechsel (s. in folgenden Abschnitt) gibt es nur wenige Regelungen eines Optionsanspruchs:

\section{Anspruch auf Erwerb einer StA (positive Option)}

Der einzige multilaterale Vertrag (M 19) gewährt einen Optionsanspruch für die StA des Aufenthaltsstaates, womit die bisherige StA endet (Art. 1 II 2), wie im vorigen Abschnitt schon erwähnt. Eine bilaterale Vereinbarung (Nr. 177) verpflichtet Flüchtlinge aus Guinea-Bissau, die 1963-1974 nach Senegal gekommen waren, für eine der beiden StA'en zu optieren, falls sie im Lande bleiben wollten.

Ein echter bilateraler StA-Vertrag (Nr. 115) gewährte den im anderen Staat wohnenden StA'en ein Optionsrecht für die dortige StA, wenn sie selber oder ein Elternteil dort geboren waren, wenn sie seit 5 Jahren dort wohnten bzw. vorher dort wohnten und mit einem Gebietsangehörigen verheiratet waren (Art. 1-2). Der Anspruch war aber dadurch eingeschränkt, daß der Staat aus vier Gründen (Sicherheit, Gesundheit, Moral, Strafen) widersprechen konnte (Art. 4).

Nr. 150: Ein einseitiges Optionsrecht war vorgesehen für Minderjährige in Zentralafrika geborene Kinder von Kamerunern: die Eltern konnten für Zentralafrika optieren, wenn ein Elternteil dort geboren war, aber seit 5 Jahren in Kamerun wohnte (Art. 6). Für den umgekehrten Fall fehlt eine Regelung.

Das von StA'en des anderen Staates adoptierte oder seit 5 Jahren erzogene Kind kann 6 Monate vor seiner Volljährigkeit für die StA dieses anderen Staates optieren (Art. 8). Minderjährige, auf die ein Wiedererwerb der StA durch die Eltern sich erstreckt, können 6 Monate vor Volljährigkeit diese StA ausschlagen und für die frühere re-optieren (Art. 10).

Da Ceylon die dortigen Inder ${ }^{32}$ als indische StA'e, Indien sie aber als staatenlos ansah, wurde ihnen durch Vertrag ein Wahlrecht für die indische o. ceylonesische StA gewährt (Nr. 55), was durch einen weiteren Vertrag noch präzisiert wurde (Nr. 62). Da die Option für Indien eine Umsiedlung zur Folge hatte, ist dies erst im nächsten Abschnitt zu behandeln.

32 S. SGS Bd. 34, S. 73-75; SGS 26, S. 47-49. 


\section{Anspruch auf StA-Entlassung (negative Option)}

Nur ein einziger StA-Vertrag (Nr. 17) gewährte britischen StA'en in Tunesien einen Anspruch auf Entlassung aus der französischen StA: Sie mußten vor 1921 in Tunesien geboren sein. Waren sie später dort geboren, so mußte mindestens auch ein Eltern- und Großelternteil (außer dem väterlicherseits) dort geboren sein. War kein Großelternteil dort geboren, so bestand der Anspruch nur für unter 22 Jahre alte, die noch keine Rechte ausgeübt hatten. Erleichterungen gab es bei britischem Wehrdienst. Frankreich verpflichtete sich, den Betreffenden nicht statt der französischen die tunesische StA aufzuerlegen.

\section{Umsiedlung und StA-Wechsel}

Seit der unglückseligen Austreibung der seit über 2000 Jahren in Kleinasien lebenden Griechen durch Abkommen von 1923 hat es zahlreiche Umsiedlungsverträge ${ }^{33}$ gegeben, insb. als Deutschland die deutschen Minderheiten "heim ins Reich" holte und sie in dem völkerrechtswidrig annektierten Korridor ansiedelte. Die einzige dieser Aktionen, die rückgängig gemacht werden konnte, betraf Südtirol ${ }^{34}$ (M 23; Nr. 24 u. 40). Nach 1945 gab es Umsiedlungsverträge, außer bei Ceylon, nur in Osteuropa (10 Verträge). Davon sind zwei Repatriierungsverträge Posen-CSR nie veröffentlicht worden (Nr. 9 u. 30), so daß diese inhaltlich nicht einzuordnen sind. Nicht hierher gehören die Vereinbarungen über Familienzusammenführungen, die die Bundesrepublik insb. mit Polen, Rumänien und der SU abschloß: darin ist die StA nicht geregelt; es erfolgt meist stillschweigend eine Entlassung. Ebensowenig ist die StA in dem bulgarisch-türkischem Umsiedlungsabkommen v. 1968 geregelt.

\section{1) Automatischer StA-Wechsel bei Grenzübertritt}

Die Option für die freiwillige Umsiedlung völkischer Minderheiten wurde im Augenblick des Grenzübertritts auch für die StA wirksam. Es trat ein automatischer StAWechsel ein. Dies galt für Tschechoslawaken in Ungarn (Nr. 11), Polen in Jugoslawien und der SU (Nr. 10 u. 80), Italienern in Jugoslawien (Nr. 174). In letzterem Vertrag erkannte Italien an, daß die Betreffenden nie die italienische StA verloren hatten, während Jugoslawien diese italienische StA erst mit dem Grenzübertritt anerkannte. Bei Nr. 80 war Besitz der polnischen StA und polnische oder jüdische Volkszugehörigkeit am 17. 9. 1939 Voraussetzung.

Eine Zwangsumsiedlung gab es bei Nr. 11: In gleicher Zahl wie Tschechoslowaken freiwillig aus Ungarn umgesiedelt wurden, konnte die CSR solche Ungarn, denen es 1945 
diese StA entzogen hatte, zwangsumsiedeln. Beim Grenzübertritt erkannte Ungarn den Verlust der csl. StA und den Besitz der ungarischen an.

\section{2) Entlassung aus StA auf Antrag}

In drei Verträgen der SU über wechselseitige Umsiedlungen mit der CSR (Nr. 7 u. 12) und Polen (Nr. 8) wurde die Option für Umsiedlung und StA-Wechsel erst mit der Genehmigung des Wohnsitzstaates wirksam. Sie wurden also bereits vor dem Grenzübertritt ausgebürgert. Daher wurde die Genehmigung bei Nr. 12 nur erteilt, wenn die neue StA zugesichert war. Bei Nr. 7 u. 8 besaßen die Tschechoslowaken bzw. Polen in der SU noch die csl. bzw. poln. StA, so daß lediglich die außerdem nach Auffassung der SU noch besessene sowjetische StA durch Ausbürgerung verlorenging. In den umgekehrten Fällen erfolgte hier aber ein echter StA-Wechsel mit Ausbürgerung im einen und Einbürgerung im anderen Staat. In allen drei Fällen bestand nach der Genehmigung Umsiedlungspflicht. In einem Vertrag über einseitige Umsiedlung garantierte Osterreich die Aufnahme, womit eine Entlassung aus jugoslawischer StA ermöglicht wurde (Nr. 56). In den Abkommen Ceylons mit Indien von 1954 (s. o.) war noch keine Umsiedlung vorgesehen. Diese wurde erst 10 Jahre später vereinbart (Nr. 134), wieder 10 Jahre später erweitert (Nr. 163 u. 166) und 12 Jahre später vorläufig abgeschlossen (Nr. 201). Hier handelte es sich wieder um Umsiedlungen in eine Richtung: Indien verpflichtete sich, 600.000 Personen aus Ceylon aufzunehmen und einzubürgern. Es war eine Zwangsumsiedlung ("Repatriierung"). Ceylon bestimmte daher in einem Gesetz zur Ausführung des Umsiedlungsabkommens von 1964: Von Indien als StA'e Anerkannte sind zur Repatriierung verpflichtet und können notfalls ausgewiesen werden.

\section{Staatsangehörigkeit und Familie ${ }^{35}$}

\section{Familieneinheit}

Ein Bekenntnis zum früher allgemein geltenden Prinzip der Familieneinheit ${ }^{36}$ in der StA findet sich, abstrakt oder konkret, nur noch in 5 Umsiedlungsverträgen der vierziger und fünf ziger Jahre in Osteuropa.

Es soll weitestmöglich "die Einheit der Familie" u. a. berücksichtigt werden (Nr. 41, Art. 6 II ) oder "der Grundsatz der Wiederherstellung bzw. Aufrechterhaltung der einheitlichen StA der Familie" beachtet werden (Nr. 28, Art. 8). In Umsiedlung und damit StAWechsel werden konkret einbezogen:

35 Nicht berücksichtigt sind hier die Doppelstaaterverträge des Ostblocks, für die kraft Sachzusammenhang die Familienfragen oben mit behandelt wurden.

36 Neuerdings: L. Diez-Picazo, El principio de unidad juridica de la familia y la nacionalidad, in: Anuario de derecho civil 1983, S. 691-701. 
Ehefrau (Ehemann), Kinder, Mutter, Vater, Adoptivkinder, weitere mitwohnende Verwandte ohne Rücksicht auf Abstammung (Nr. 8, Art. 4), Frau, Kinder, Mutter, Vater, Enkel, Mündel, betreute Personen und andere Hausangehörige mit gemeinsamen Haushalt (Nr. 12, Art. 10), Ehegatten, Kindern, Eltern, soweit mitwohnend, auch ohne polnische Volkszugehörigkeit und ohne polnische StA 1939 (Nr. 80, Art. 4); Familienangehörige ohne polnische Volkszugehörigkeit, aber sowjetischer StA verlieren diese nur bei Option f. d. poln. StA (Nr. 80, Art. 10).

\section{Automatischer StA-Wechsel bei Ehe}

Dies sieht noch M 20 vor, außerdem nicht bei Staatenlosigkeit des Mannes und nicht bei ausdrücklicher Beibehaltungserklärung der Frau (Art. 1). Wird dem Mann später seine StA entzogen, so erwirbt die Frau ihre frühere StA automatisch zurück (Art. 2). Nach Beendigung der Ehe kann sie in ihre Heimat zurückkehren und ihre frühere StA durch Antrag wiedererwerben, womit sie automatisch die durch die Ehe erworbene StA wieder verliert (Art. 3).

M 3 schließt jeden automatischen Wechsel hier aus, gibt der Frau aber einen Einbürgerungsanspruch (mit Widerrufsvorbehalt). M 9 (Art. 9 I) bestätigte die Ablehnung jeder Automatik.

In bilateralen Verträgen sind verschiedene Lösungen gewählt:

Nr. 16: Grundsätzliche Automatik, falls nicht in 6 Monaten für Beibehalt optiert wird oder ein Ausweisungsbefehl vorliegt oder die Regierung dem Erwerb widerspricht (Art. 1). Heiratet eine belgisch-französische Doppelstaaterin den Angehörigen eines Vertragsstaates, dann gilt nur die StA des Eheschließungsortes (Art. 4).

Nr. 65: Grundsätzlich keine Automatik, wohl aber wenn ein Ehepartner freiwillig die StA des anderen beantragt (Art. 10).

Nr. 67: Automatik bei Ehen vor 1955, aber jetzt Wahlrecht der Frau auch ohne Manneszustimmung (Art. 11) und Wiedererwerb nach Eheauflösung (Art. 14). Bei Ehen nach 1955 Automatik nur, wenn die Frau eines Vertragsstaates im anderen Staat heiratet, aber auch dann Beibhaltungserklärung möglich. Bei Ehen nach 1955 in einem dritten Staat keine Automatik, es sei denn Antrag auf Erwerb (Art. 12-13).

Nr. 150, Art. 7: Eine Kamerunerin, die einen Zentralafrikaner heiratet, erwirbt automatisch dessen StA, falls sie diese nicht bei der Eheschließung ausschlägt. Im umgekehrten Fall erwirbt eine Zentralafrikanerin, die einen Kameruner heiratet, nicht automatisch dessen StA, kann aber bei der Eheschließung dafür optieren. 


\section{Erstreckung eines späteren StA-Erwerbs auf den Ehegatten}

Nur in 2 Verträgen findet sich eine automatische Erstreckung (Nr. 28 u. 34). Bei Nr. 28 erfolgt die Erstreckung auf den zusammenlebenden Ehegatten (also ev. auch den Ehemann!), innerhalb von 3 Monaten ist aber Re-option möglich gewesen (Art. 9). Bei Nr. 34 war Re-option erst nach Auflösung der Ehe möglich (Art. 10). In den meisten Fällen wird ausdrücklich hervorgehoben, daß eine Option sich nicht auf die Ehef rau erstreckt (M 23, Art. 19 II, 20 IV, Anlage VI, Art. 6 II; Nr. 42, Art. 3; Nr. 70, Art. 5; Nr. 115, Art. 3) und es wird auch betont, daß Ehefrauen selber optieren müssen (Nr. 65, Art. 1) oder daß sie durch Zustimmung in die Option des Mannes einbezogen werden können (Nr. 106, Art. 11). Nr. 109 gewährt der Frau ausdrücklich einen Anspruch auf Erstreckung (Art. 4 [3-6]). Nr. 176 gibt auch der Frau einen eigenen Optionsanspruch (Art. 5 III). Wurde die StA eines Ehegatten durch den Vertrag geändert, so kann er innerhalb von 5 Jahren für die StA des anderen optieren, bei Eheauflösung erwirbt er automatisch die frühere StA zurück (Art. 5 VII).

\section{Originäre StA der Kinder}

Eine Ausnahme vom ius soli machen M 4 u. 6: Kinder von Konsuln und Diplomaten erwerben nicht automatisch die StA des Geburtsortes in Ländern, wo sonst ius soli gilt. Eine Ausnahme davon, daß beim ius sanguisnis sich die StA des ehelichen Kindes nach der des Vaters richtet, macht Nr. 9: das Kind erwirbt auch die StA der Mutter. Es wird also hier Doppelstaatigkeit gefördert.

Eine Ausnahme von der Regel, daß das uneheliche Kind die StA der Mutter erwirbt, macht M 20: dies gilt nur, solange die Vaterschaft nicht gesetzlich festgestellt ist; ist dies der Fall, so erwirbt ein Kind unter 18 Jahren die Vertrags-StA des Vaters und verliert die der Mutter (Art. 5).

Nr. 150: Kein ius soli für eheliche und von beiden Eltern anerkannte nichteheliche Kinder (Art. 1-2). Ius soli für Findelkinder, falls nicht während Minderjährigkeit Abstammung von StA'en des anderen Staates festgestellt wird (Art. 5). Bei Mischehen ius sanguinis nach Vater, auch bei nichtehelichen Kindern (Art. 3): nur wenn dieser bei ehelichen Kindern staatenlos ist, nach der Mutter (Art. 4).

\section{Option u. StA der Kinder}

Eine automatische ausnahmslose Erstreckung der Option des Vaters auf alle minderjährigen Kinder, haben M 20, Nr. 47, 67 u. 115, jedoch gibt es im ersteren Fall Re-option bei Volljährigkeit (ebenso bei Nr. 67 u. 70). Eine Erstreckung nur auf ledige Minderjährige findet sich bei M 23, Nr. 42, 66 u. 70.

Ersatzweise erfolgt die Erstreckung auf die StA der Mutter, wenn der Vater tot ist 
(M 23), wenn er tot oder unbekannt ist (Nr. 106), wenn er unehelicher Vater ist oder die Ehegatten in einem anderen Land getrennt leben (Nr. 176, Art. 6), wenn Getrenntleben vorliegt (Nr. 174), wenn bei Unehelichen nur die Abstammung nach der Mutter festgestellt wurde (Nr. 67, Art. 7).

Eine Erstreckung findet nicht statt, es seidenn die Eltern optieren ausdrücklich auch für ihre minderjährigen Kinder: Nr. 73, Nr. 121 (hier für Beibehaltung) u. Nr. 109 (für Kinder unter 16).

Auch Minderjährige müssen selber optieren: so verheiratete Männer ab 16 (Nr. 42 u. 70), so haben 1960/1 Geborene, wenn ein Elternteil für Zypern optierte, ebenfalls einen Anspruch darauf (Nr. 109, Sec. 4, 6).

Nr. 176 sagte: 25 Jahre lang können Kinder und Adoptivkinder bestimmter Personen für Surinam optieren (Art. 5, 3); außerhalb Surinams geborene Kinder können 5 Jahre ab der Volljährigkeit für es optieren (Art. 6, 5).

\section{E. Verschiedenes}

\section{Art. 116 I GG $^{37}$}

In keinem multilateralen Vertrag hat die BRD eine Definition des "Deutschen" nach Art. 116 I GG durchsetzen können. Sie hat nur entsprechende einseitige Erklärungen zum EWG-Vertrag und den Assoziierungsabkommen abgegeben, die die Partner nicht binden. Dagegen findet sich ein Hinweis auf Art. 116 I GG als Vertragsbestandteil in vielen bilateralen Verträgen ${ }^{37 a}$ betr. Kultur (Nr. 68, 74, 79), Freundschaft $(85,182)$, Doppelbesteuerung $(102,143)$, Auslieferung (89), Konsuln (72). Entsprechende Klauseln finden sich ferner in den oben behandelten Abmachungen über Austausch von Einbürgerungsmitteilungen.

Diese Klausel ist zunächst eine Ausnahme von der Regel, daß ein Staat nur eigene Staatsangehörige schützen darf. Die BRD kann nämlich nach Art. 116 I GG auch diejenigen Statusdeutschen, die staatenlos sind oder noch eine andere Staatsangehörigkeit besitzen, wie eigene StA'e behandeln.

Weit wichtiger aber ist folgendes: Wer am 23. 5. 1949 nach dem RuStAG die deutsche StA besaß, kann danach von der BRD als "Deutscher" beansprucht werden, d. h. auch die Bewohner der damaligen SBZ und deren Abkömmlinge. Von der DDR Eingebürgerte wird dagegen die BRD schwerlich völkerrechtlich beanspruchen können. Die Vertragspartner der BRD sind durch die Klausel auch nicht gehindert, daneben eine DDRStA anzuerkennen, also in der Regel Doppelstaatigkeit anzunehmen.

37 Vgl. Hecker, in: v. Münch, Grundgesetzkommentar, 2. Aufl., 1983, S. 1112 f.

37a Aus der Fülle dieser Verträge können hier nur einige Muster aufgeführt werden. Vgl. Makarov/v. Mangoldt, Deutsches StARecht, Einl. V Rdnrn. $162 \mathrm{ff}$. 


\section{Recht auf Staatsangehörigkeit}

Ein solches Recht auf Erwerb oder Fortbestand einer StA ist nur in multilateralen Verträgen geregelt (über Nr. 150 s. o.).

\section{1) Erwerb}

Von einem "Recht auf eine StA" sprechen ganz allgemein zwei Verträge (M 8 u. 22) und drei Erklärungen (Art. 19 der amerik. MRE v. 2. 5. 1948; Art. 15 MRE v. 10. 12. 1948 und Art. 3 der Erklärung der Rechte des Kindes v. 20. 11. 1959), während M 8 dies dahin präzisiert: "Jedes Kind hat das Recht, eine StA zu erwerben". Ob darin ein Anspruch auf Erwerb der StA des Geburtslandes (ius soli) für sonst Staatenlose gesehen werden kann (wie dies Art. 20 II vom M $22^{38}$ als Automatik ausdrücklich formuliert) ist zweifelhaft, denn das ius soli ist zwar ein lateinamerikanisches Prinzip, aber kein universelles. Nur Findelkinder würden zweifellos die StA des Fundstaates erwerben (so auch Art. 2 v. M 5). M $5^{39}$ regelt noch folgendes:

Ein staatenlos Geborener hat einen Anspruch auf die StA des Geburtslandes, falls nicht 4 Ablehnungsgründe vorliegen. Der Staat kann ihm aber auch automatisch seine StA verleihen. Unabhängig davon soll ein sonst staatenloses eheliches Kind qua Geburt die StA seiner Mutter erwerben (ebenso Art. 1 v. M 18). Kann ein Staatenloser die StA des Geburtslandes mangels Wohnsitz oder fristgemäßer Option nicht erwerben, besitzt aber ein Elternteil bei seiner Geburt diese StA, so soll er eingebürgert werden, wobei nur 3 Ablehnungsgründe zulässig sind. In einem Nichtvertragsstaat geborene Kinder erhalten einen Anspruch auf die StA desjenigen Elternteils, der die StA eines Vertragsstaates besitzt (4 Ablehnungsgründe).

Nach M 19 erwirbt jeder aus einem Vertragsstaat stammende Staatenlose die dortige StA kraft ius soli.

Zwei Treuhandabkommen (Nr. 20 u. 37) verpflichten die Treuhänder, den Eingeborenen eine eigene gebietszugehörige StA zu verleihen. ${ }^{40}$

\section{2) Bestandsgarantie}

M 5 verbietet StA-Verlust aus familienrechtlichen Gründen oder bei Erstreckungen auf Familienangehörige oder Verzicht, falls keine andere StA erworben ist. Ein automatischer Verlust bei Auslandswohnsitz ist mit der Folge der Staatenlosigkeit nur erlaubt, wenn 7 Jahre lang keine Beibehaltungserklärung erfolgte. Nur dann und bei Betrug oder

38 Ein interessantes Gutachten des Interamerikanischen Gerichtshofs für Menschenrechte vom 19. 1. 1984 befaßte sich auf Antrag Costa Ricas mit der Frage, ob von diesem geplante Änderungen des StAR's mit Art. 20 der amerikanischen Menschenrechtskonvention vereinbar seien, Was der Gerichtshof bejahte (EuGRZ 1985, S. 502-511).

39 Zur Konvention: AFDI 1962, S. 528-555 u. ICLQ 1962, S. 1073-1096.

40 Zu Nr. 37: SGS 37, S. 167. 
Untreue ist eine entsprechene Entziehung möglich. Aus rassischen, ethnischen, religiösen oder politischen Gründen darf die StA nie entzogen werden. M 22 verbietet jede Zwangsausbürgerung und das Bestreiten des Rechts auf StA-Wechsel, was schon Art. 19 der amerik. MRE und Art. 15 II MRE als Programm aufgestellt hatten.

\section{Zwischenstellung zwischen In- und Ausländern}

Bilaterale Verträge sehen vor, daß die Angehörigen eines Staates im anderen besser als sonstige Ausländer gestellt werden, so bei Gastarbeitern ( $\mathrm{Nr}$. 77), hinsichtlich von Papieren (Nr. 139) oder Aufenthalt (Nr. 138). Besonders ausführlich Nr. 155 (Ausweise; kein StA-Verlust bei Rechtsausübung usw.).

Ein ähnlicher Sonderstatus wurde in Dekolonialisierungsfällen vereinbart:

Die Bewohner der Insel St. Marie de Madagaskar, die besonders französiniert waren, durften trotz malgachischer StA in Frankreich noch Bürgerrechte ausüben und ihr Personalstatut behalten (Nr. 107). Ähnliches galt für Algerien (Nr. 120) und Optanten in Franz. Indien (Nr. 126). Die "Brit. Dep. Terr. Citizens" in Hongkong behalten trotz alleiniger chines. StA einen Sonderstatus: sie können einen brit. Paß erhalten, der außerhalb Chinas dipl. Schutz verleiht und werden durch diese Option somit eine Art Schutzgenossen (Nr. 200).

Personen, die nicht die StA von Brunei besaßen, durften bei der Unabhängigkeit wohnen bleiben und bekommen Pässe als quasi-Schutzangehörige Bruneis; sonst sind sie auf normale Einbürgerung verwiesen (Nr. 185).

Die Bewohner der Nordmarianen erwerben, wenn die Treuhandverwaltung endet, automatisch die volle Citizenship der USA; sie können aber dafür optieren, bloße US-nationality ohne Citizenship zu erwerben (Nr. 173), womit sie eine Art Schutzgenossen werden. ${ }^{41}$

\section{Regelung der Nichtregelung der StA}

\section{1) Austausch von Einbürgerungsmitteilungen}

In zwei multilateralen (M 14, Art. 6; M 15, Art. 5) und einem bilateralen Vertrag (Nr. 180) findet sich hierbei die Klausel, daß StA-fragen dadurch unberührt bleiben, also insbes. kein StA-Verlust durch E:..bürgerung unterstellt wird. 


\section{2) Wehrdienst von Doppelstaatern}

Hier findet sich in fast allen Verträgen eine Klausel, daß StA-fragen dadurch nicht berührt werden. Wenn also ein Staat Wehrdienst im Ausland als Grund für den StA-Verlust ansieht, dann tritt dieser auch ein, wenn dieser Wehrdienst vom inländischen Wehrdienst auf Grund des Vertrages befreit. Das Haager-StA-Protokoll von 1930 hatte in Art. 1 II sogar ausdrücklich vorgesehen, daß eine solche Befreiung den StA-Verlust zur Folge haben könne. Weitaus die meisten solcher Verträge hat Frankreich geschlossen: Nr. 18, Art. 6; Nr. 26 u. 27, Art. 6; 29, Art. 4; 31, Art. 5; 32, Art. 7; 35, Art. 9; 54, Art. 9 I; 94, Art. 7 I; 101, Art. 8; 123, Art. 11; 128, Art. 8; 147, Art. 9; 172, Art. 12; 196, Art. 7 (es bleibe hinsichtlich der StA ausschließlich beim nationalen Recht). Außerdem Italien: Nr. 60, Art. 7; 71, Art. 8; 114, Art. 10; 170, Art. 12; 195, Art. 16. Argentinien: Nr. 26 a, Art. 3; 124, Art. 3; 127, Art. 6; 129, Art. 4; 191, Art. 3. BelgienNiederlande: Nr. 59, Art. 13. In zwei allgemeinen Wehrpflichtabkommen Großbritanniens findet sich dieselbe Klausel (Nr. 61, Art. 10; Nr. 131, Art. 4 II).

\section{3) Ostverträge und Neuguinea}

Eine dreifache Stufung von Ausklammerungen der StA findet sich in den Ostverträgen der Bundesrepublik: Im Warschauer Vertrag wurde die StA-frage zwar in den Verhandlungen angesprochen, aber dann nur in dem einseitigen Schlußkomunique der BRD wie folgt erwähnt:

"Wir haben bei Abschluß der Verhandlung betont, daß durch den Vertrag niemandem

Rechte verloren gehen, die ihm nach unseren geltenden Gesetzen zustehen (z. B. StA)".

(Bulletin der BR v. 8. 12. 1970, S. 1819).

Im Grundvertrag (Nr. 162), der an der StA-frage zu scheitern drohte, wurde in einseitigen Protokollvermerken beider Seiten erwähnt, daß StA-fragen nicht geregelt wurden (BRD) bzw., daß der Vertrag die StA-frage erleichtere (DDR). Beide Seiten haben in ihren Gesetzblättern nur den eigenen Vermerk abgedruckt, den der anderen Seite aber ignoriert.

Im Prager Vertrag (Nr. 165) schließlich wurde im Vertragstext selber erklärt, daß StAfragen ausgeschlossen worden seien.

In einer offiziellen Anmerkung zu Nr. 34 vereinbarten die Vertragspartner, daß über die StA der Bewohner von Neuguinea "in den vorstehenden Artikeln nichts bestimmt" sei, solange dies nicht indonesisch werde.

\section{Atypische Sonderfälle}

Eine gemischte StA-Kommission sollte Empfehlungen bei Streitfällen abgeben (Nr. 19), sie_wurde aber schon ein Jahr später wieder aufgelöst. ${ }^{42}$ Wer "ressortisant cameroun"

42 S. VRU 1971, S. 79 
war, gelte als Kameruner nach Art. 2 des Statuts für Kamerun vor der Unabhängigkeit; eine - besondere - nie abgeschlossene - Konvention solle die Stellung anderer Kameruner mit franz. StA und deren dipl. Schutz regeln sowie Fragen der Staatenlosen u. Doppelstaater (Nr. 98).

In M 11 stimmten die 3 Westmächte zu, daß West-Berlin u. a. auch das StA-Recht der BRD übernehmen könne. 


\section{Anlage}

\section{Liste der Verträge}

$\mathrm{x} \quad=$ in den UNTS veröffentlicht. Von den über 200 bilateralen Verträgen sind nur 78, also nicht einmal die Hälfte, dort veröffentlicht; von den 22 multilateralen dagegen 14. Nr. 173b und Nr. 175 sind für Bd. 1096 und 1097 vorgesehen, die aber noch nicht erschienen sind,

Klammern $=$ Datum des Inkrafttretens. Liegt dies im gleichen Jahr, so ist das Jahr nicht wiederholt. Fallen Vertragsschluß und Inkrafttreten zusammen, so ist dies durch ein Gleichheitszeichen ausgedrückt.

Datum: $\quad$ Bei Notenwechseln gilt als Vertragsdatum das der letzten Note.

Vertrags-

änderungen: Diese sind stets nur beim Hauptvertrag erwähnt.

Reihen- Die Vertragspartner werden in alphabetischer Rangordnung nach den Deutschen folge: $\quad$ Staatennamen aufgeführt. Dabei gilt BRD als Deutschland, CSR als Tschechoslowakei, USA als Vereinigte Staaten.

Artikel: $\quad$ Bei Nicht-StA-Verträgen sind die Artikel mit einer StAB angegeben.

Fundstellen: für Europa: Werkhefte 25 (1974)

für Amerika: Das Staatsangehörigkeitsrecht von Amerika (1984)

für Afrika, Asien und Australien: Sammlung geltender Staatsangehörigkeitsgesetze

\section{A. Multilaterale Verträge}

(im Text abgekürzt durch $\mathrm{M}$ )

\section{A. Universale}

1) $x$ 28.7.1951(22.4.54) Rechtstellung der Flüchtlinge, Art. 34 (x Prot. v. 31. 1. 67)

2) $x$ 29.9.1954(6.6.60) Recht der Staatenlosen, Art. 32

3) $x$ 29.1.1957(11.8.58) StA der Ehefrau

4) $x$ 18.4.1961(24.4.64) Fak.protokoll: StA Diplomatenkinder

5) 30.8.1961(13.12.75) Verringerung der Staatenlosigkeit

6) $x$ 27.4.1963(19.3.67) Fak.protokoll: StA der Kinder v. Konsuln

7) $x$ 7.3.1966(4.1.69) Rassen-Diskriminierung, Art. 1 u. 5

8) 16.12.1966(23.3.76) Menschenrechte: Politischer Pakt, Art. 24 III

9) 18.12.1979(3.9.84) Frauen-Diskriminierung, Art. 9

B. Regionale: Europa

10) $\mathrm{x}$ 21.12.1950(1.1.51) Nordische Staaten: Erleichterung StA-Erwerb

11) $x$ 26.5.1952(5.5.55) 3 Westmächte: Berlinbrief Nr. 1

12) $\mathrm{x}$ 3.3.1956(=) Nord. Staaten: Wehrpflicht u. StA

13) $x$ 25.3.1957(1.1.58) EWG, Schlußakte, Einseitige Erklärung d. BRD

14) $x$ 6.5.1963(28.3.68) Doppelstaater und Wehrpflicht (CE Nr. 34) (Prot. 24. 11. 77)

15) $x$ 10.9.1964(30.9.65) Auskunft StA-Information (CIEC Nr. 8) 
16) $\times$ 24.4.1967(26.4.68)

17) $\times 15.1 .1969(1.1 .69$ !)

18) $\quad 13.9 .1973(31.7 .77)$

Adoption (CE Nr. 58), Art. 11

Nordische Staaten: Erleichterung StA-Erwerb

Verringerung der Staatenlosigkeit (CIEC Nr. 13)
C. Regionale: Ubersee

19) 23.9.1952(5.1.55)

20) $\quad 5.4 .1954$

(Noch nicht) Arabische Liga: Einige Bestimmungen über StA

21) 1.12.1965(nie) Westafrika: Einführung v. Doppelstaatigkeit

22) 22.11.1969(18.7.78) American Convention on Human Rights, Art. 20

D. Friedensvertrag

23) $\quad$ 10.2.1947(16.9.)

Friedensvertrag der Alliierten mit Italien, Art. 19, 20, 30, 36; Anlage IV v. 5. 9. 1946, Art. 3 a; Anlage VI, Art. 6

\section{B. Bilaterale Verträge}

\section{5}
1) $\quad 14.2 .1945(=)$
2) 25.2.(10.6.40!)
3) $x$ 2.4.(5.4.)
4) $x$ 10.5.(11.5.)
5) $x$ 23.5.(12.6.)
6) $x$ 7.6.(11.6.)
7) $x$ 29.6.(30.1.46)
8) 6.7 .
9) $\quad 21.9$.

\section{6}

10) $\mathrm{x}$ 2.1.(=)

11) 27.2.(15.5.)

12) $\quad 10.7 .(=)$

13) $\mathrm{x}$ 4.11.(30.11.48)

14) $\times$ 17.11.(=)

15) 7.12 .

\section{7}

Deutschland-Ungarn, Waffen-SS, Art. 13

Frankreich-Italien, Noten betr. Außerkrafttreten der Tunisverträge (m. StAB) v. 1896

Ecuador-USA, Noten betr. Wehrdienst

USA-Venzuela, Noten betr. Wehrdienst

Peru-USA, Noten betr. Wehrdienst

Chile-USA, Noten betr. Wehrdienst

SU-CSR, Karpatho-Ukraine, Zusatzprotokoll Art. 2

Polen-SU: Umsiedlung u. StA

Polen-CSR: Repatriierung

Polen-Jugoslawien: Umsiedlung nach Polen

CSR-Ungarn: Umsiedlung

SU-CSR: Umsiedlung aus Wolhynien

China-USA: Handel, Art. 14

Frankreich-Thailand: Noten, StA in wiedergewonnenen Gebieten Libanon-Türkei: Optantenprotokoll, Fristverlängerung: 16. 12. 54 und 28. 1. 1957

Belgien-Frankreich: StA der Ehefrau

Frankreich-Großbritannien: Brit. StA'e in Tunesien

Brasilien-Frankreich: WDS im 2. Weltkrieg 

19) 8.3 .
20) $x$ 2.4.(18.7.)
21) $\quad$ 16.6. $(=)$
22) $\times$ 17.10.(4.1.48)
23) $\quad 21.11 .(=)$
24) 22.11.(=)

1948
Polen-USA: Errichtung einer StA-Kommission Treuhandabkommen mit USA über TTPI, Art. 11 Frankreich-Monaco: StA Burma-Großbritannien: Unabhängigkeit, Art. 5 Bulgarien-Jugoslawien: Grenzfragen, Art. 25-26 Italien-Osterreich: Optanten Südtirol

Italien-USA: Handel, Art. 13 (Wehrpflicht) Frankreich-USA: Wehrdienst von Doppelstaatern, im folg.: WDS Argentinien-Spanien: Wehrdienst, Art. 3 Frankreich-USA: WDS (x 31. 12. 52 Ergänzung)

1949

$\begin{array}{lll}28) & & 10.2 .(19.6 .) \\ 29) & 30.3 .(18.1 .50) \\ 30) & & 6.5 . \\ 31) & \times & 22.6 .(31.10 .) \\ 32) & \times & 29.8 .(1.6 .51) \\ 33) & \times & 31.8 .(=) \\ 34) & \times & 2.11 .(27.12 .) \\ 35) & \times & 21.12 .(12.5 .56)\end{array}$

1950

36) $\times$ 21.1.(14.9.)

37) 27.1.(8.1.52)

38) $\quad 3.3 .(5.1 .51)$

39) $x$ 25.3.(1.4.)

40) 28.3.(=)

41) 23.12.(=)

\section{1}

42) $\mathrm{x} \quad 2.2 .(9.6 .52)$

43) $\quad 13.2$.

44) 29.11.(9.7.53)

45) 20.12 .

\section{2}

46) $\quad 18.1$.

$47 \quad 27.3$.

48) $\times$ 28.4.(5.8.)
Rumänien-Ungarn: StA

Frankreich-Luxemburg: WDS

Polen-CSR: Repatriierung aus Ost-Slowakei

Dänemark-Frankreich: WDS

Belgien-Frankreich: WDS

Griechenland-Italien: Ausführung des FV, Art. 29

Indonesien-Niederlande: Aufteilung der StA'en

Frankreich-Großbritannien: WDS

Irland-USA: Handel, Art. 3 II u. Protokoll Ziff. 3

Treuhandabkommen m. Italien über Somaliland: Annex Art. 2

Frankreich-Saarland, Allg. Konv., Art. 5

Jugoslawien-USA, Noten: Visa f. Doppelstaater

Italien-Osterreich: Reoptanten in Südtirol

Italien-Jugoslawien: Optionen nach FV

Frankreich-Indien, Abtretung v. Chandernagore, Art. 2-4

Australien-BRD: Austausch von Einbürgerungsmitt., im folg. AEM

BRD-Frankreich: Auslief erung, Art. 2 (2)

BRD-Luxemburg: AEM (Erg.: 1953 u. 1954)
BRD-Niederlande: AEM (Erg.: 1954)

Italien-Jugoslawien: Optionen in Mahovljani

Japan-Taiwan: FV, Art. 10 
49) $\quad 13.6$.

50) $\quad 19.8$.

1953

51) $\quad 26.9$.

52) 8.10 .

53) 18.12.(13.1.55)

54) $\times$ 28.12.(11.4.57)

\section{4}

55) 18.1.(13.2.)

56) 12.3 .

57) 17.5.

58) $\quad 1.6$.

59) $\mathrm{x}$ 9.6.(19.8.55)

60) $x$ 15.7.(3.5.56)

61) 31.7.(25.9.59)

62) 10.10.(=)

63) 4.11.(=)

64) $\times$ 18.12.(10.2.56)

1955

65) 22.4.(20.1.60)

66) 3.6.(31.8.)

67) 16.8.(=)

1956

68) $\quad 8.2 .(9.12 .57)$

69) $\times$ 22.5.(31.7.)

70) 28.5.(=)

71) $\mathrm{x}$ 4.6.(28.10.59)

72) 30.7.(28.12.)

73) $\quad 20.9$.

74) $\mathrm{x}$ 24.9.(28.8.58)

75) $16.10 .(1.10 .56 !)$

76) $\times 27.10 .(1.1 .57)$

77) 28.11.

78) 20.12.(=)
BRD-Kanada: AEM

BRD-Pakistan: AEM (Erg.: 1955)

BRD-Griechenland: AEM

BRD-Malaya: AEM

BRD-Jugoslawien: AEM

Frankreich-Italien: WDS

Indien-Sri Lanka: Umsiedlung, Art. 4-7

Jugoslawien-Osterreich: Umsiedlung nach Osterreich

BRD-Japan: AEM

BRD-Irak: AEM

Belgien-Niederlande: WDS (Änd.: x 25. 10. 62)

Dänemark-Italien: WDS

Chile-Großbritannien: Wehrdienst, Art. 10 (StA unberührt)

Indien-Sri Lanka, Statement, Ziff. 2-7

BRD-Iran: Wiederanwendung Niederlass.vertrag 1929 m. Schlußprot. Ziff. 2 (Einbürgerung)

Italien-Jugoslawien: Brief A zum FV-Ausführungsvertrag

China-Indonesien: Doppelstaater (Noten dazu v. 3. 6.)

Frankreich-Tunesien: Personenstand, mit Protokoll 1

Frankreich-Vietnam: StA

BRD-Italien: Kultur, Art. 5

Jugoslawien-SU: Doppelstaater (Fristverläng.: 29. 8. 57)

Frankreich-Indien: Abtretung Franz.Indien, Art. 4-8

Chile-Italien: WDS

BRD-Großbritannien: Konsular, Art. 1 (4)

China-Nepal, Grenze, Art. 14

Belgien-BRD, Kultur, Art. 3

Dänemark-BRD: Wiederanwendung Vertrag v. 1939 AEM

BRD-Frankreich: Saar

Belgien-Spanien: StA-Nachweis v. Gastarbeitern

BRD-Peru: AEM 

79) 14.2.(10.10.)
80) $\times$ 25.3.(10.7.)
81) $\times$ 24.8.(15.1.58)
82) $\mathrm{x}$ 4.9.(3.3.58)
83) $\times$ 18.9.(29.4.58)
84) $\mathrm{x} \quad 5.10 .(21.7 .58)$
85) 21.11.(19.11.61)
86) $\times$ 12.12.(28.3.58)
87) $\mathrm{x}$ 16.12.(5.2.58)
88) 23.12.(3.6.60)

1958
BRD-Japan: Kultur, Art. 10

Polen-SU: Repatriierung (24.6.58 Frist verlängert)

SU-Ungarn: Doppelstaater

Rumänien-SU: Doppelstaater

Albanien-SU: Doppelstaater

SU-CSR: Doppelstaater

BRD-Italien: Prot. zu Freundschaftsvertrag, Ziff. 2 u. 17

Bulgarien-SU: Doppelstaater

Korea (Nord)-SU: Doppelstaater

BRD-Domin.Rep.: Prot. zu Freundschaftsvertrag, Ziff. 2
Belgien-BRD: Auslieferung, Art. 4 m. Prot. Ziff. 1

Polen-SU: Doppelstaater

Chile-Spanien: Doppelstaater (dazu Noten v. 23. 6. 58)

Bulgarien-Ungarn: Doppelstaater (m. Protokoll betr. Einbürgerung)

BRD-Ecuador: AEM

Frankreich-Schweiz: WDS (dazu Prot. v. 1961)

Mongolei-SU: Doppelstaater

Brasilien-Italien: WDS

BRD-Osterreich: AEM

Frankreich-Kamerun: Zusammenarbeit, Art. 4

1959

\footnotetext{
99) $\quad 16.5 \cdot(10.2 .60)$

100) 25.6.(10.3.60)

101) $\times$ 30.6.(7.5.62)

102) $\times$ 21.7.(4.11.61)

103) $\times$ 24.9.(24.12.)
}

\section{0}

Peru-Spanien: Doppelstaater

Paraguay-Spanien: Doppelstaater

Frankreich-Israel: WDS (m. Noten; u. Erg. x 22. 12. 66)

BRD-Frankreich: Doppelbesteuerung, Art. 21 (2)

Bulgarien-Rumänien: Doppelstaater

\begin{tabular}{|c|c|}
\hline 104) & 9.2 . \\
\hline 105) & 18.3.(21.10.61) \\
\hline 106) & x 8.4.(1.8.63) \\
\hline 107) & 27.6.(18.7.) \\
\hline 108) & 9.8 \\
\hline 109) & 16.8. \\
\hline 110) & x 1.10 \\
\hline 111) & x 4.11.(19.3.61) \\
\hline 112) & x 10.11.(=) \\
\hline 113) & 24.12.(=) \\
\hline
\end{tabular}

BRD-Panama: AEM

BRD-Griechenland: Niederlassung, Prot. Ziff. 4 (WDS)

BRD-Niederlande: Grenze, Art. 11

Frankreich-Madagaskar: Status St. Marie

Brasilien-Portugal: Doppelstaater

Großbritannien-Türkei: Zypern, Art. 6 u. Annex D

Burma-Chile: Grenze, Note dazu Ziff. 1

CSR-Ungarn: Doppelstaater

Dänemark-BRD: AEM

China-Indonesien: Ausführung v. 1955 

114) $\mathrm{x} 24.1 .(18.10 .62)$
Italien-Niederlande: WDS
115) 14.6.(29.6.65)
Libyen-Tunesien: Option
116) $\mathrm{x}$ 5.7.(3.2.62)
117) 25.7.(26.3.62)
Polen-Ungarn: Doppelstaater
118) 28.7.(25.1.62)
Nicaragua-Spanien: Doppelstaater
119) $\mathrm{x}$ 12.10.(31.3.64)
Guatemala-Spanien: Doppelstaater
Bolivien-Spanien: Doppelstaater

\section{2}

120) $x$ 19.3.(3.7.)

121) $\quad 14.8$.

122) 13.9.(=)

123) $\quad 12.10 .(5.11 .64)$

124) $\times$ 28.11.(=)

\section{3}

125) $\mathrm{x}$ 2.1.(19.8.)

126) 16.3.(16.6.)

127) $\mathrm{x}$ 8.5.(5.11.)

128) $x$ 6.6.(3.2.65)

129) 11.6.(2.8.67)

130) $x$ 17.7.(=)

131) $\times$ 12.9.(22.3.67)

\section{4}

132) 4.3.(24.12.)

133) 8.6.(21.5.65)

134) $31.10 .(=)$

135) 27.11.(2.12.)

1965

136) $\times$ 31.3.(30.1.66)

137) $x$ 17.5.(20.5.66)

138) 22.6 .

1966

139) 4.3.

140) 15.6.(25.4.67)

Argentinien-Belgien: WDS
Algerien-Frankreich: Evian, Allg. Erklärung u. Garantieerklärung je Kap. II

China-Nepal: Grenze, Noten, Ziff. I

Chile-BRD: AEM

Belgien-Frankreich: WDS

Argentinien-Dänemark: WDS

SU-Ungarn: Doppelstaater

Frankreich-Indien: Ausführung von 1956, Ziff. I, 1-2

Argentinien-Finnland: WDS

Dänemark-Frankreich: WDS

Dänemark-Österreich: AEM

Argentinien-Großbritannien: Wehrpflicht (wichtig f. Falklandinseln!)

Ecuador-Spanien: Doppelstaater

Costarica-Spanien: Doppelstaater

Ceylon-Indien: Ceylon-Inder

Italien-Jugoslawien: Optionen

Polen-SU: Doppelstaater

Polen-CSK: Doppelstaater

Japan-Korea (Süd): Status v. Koreanern in Japan

Columbien-Ecuador: Status im anderen Staat

Honduras-Spanien: Doppelstaater 
141) $\mathrm{x}$ 6.7.(19.1.67)

142) 10.9.(28.1.67)

1967

143) $\times$ 11.4.(30.7.69

144) $x$ 15.11.(=)

1968

145) 15.3.(22.1.69)

1969

146) 4.1.(13.5.)

147) 9.4.(1.8.70)

148) $\mathrm{x}$ 11.4.(13.2.70)

149) 14.4.(23.3.71)

150) 24.7.(nie)

151) $\mathrm{x}$ 17.12.(8.7.70)

1970

152) 23.4.(26.11.72)

153) $\times$ 26.11.(14.11.75)

1971

154) $x \quad 1.7 .(=)$

155) $x$ 7.9.(22.4.72)

156) $\quad 1.10 .(11.5 .72)$

157) 29.10.(12.9.74)

1972

158) x 21.1.(15.9.)

159) 7.2.(25.9.)

160) $\times$ 31.5.(6.7.73)

161) $\times$ 31.5.(6.7.73)

162) 21.12.(21.6.73)
Bulgarien-SU: Doppelstaater (m. Protokoll betr. Art. 5 v. 12. 12. 57)

Argentinien-Vatikan: Konkordat, Art. 5 (2)

Belgien-BRD: Doppelbesteuerung, Art. 24

Großbritannien-Oman: Kuria Muria, Art. 3

Dominik.Rep.-Spanien: Doppelstaater

Marokko-Spanien: Ifni, Art. 3 u. Prot. Art. 1

Frankreich-Spanien: WDS

DDR-SU: Doppelstaater

Argentinien-Spanien: Doppelstaater

Kamerun-Zentralafrika: StA

DDR-Ungarn: Doppelstaater

BRD-Spanien: Niederlassung, Art. 6 (5) (WDS)

BRD-Jugoslawien: Auslieferung, Art. 6 II

Argentinien-Großbritannien: Falkland-Inseln

Brasilien-Portugal: Gleichberechtigung, Art. 2, 4, 13

Bulgarien-DDR: Doppelstaater

Argentinien-Italien: Doppelstaater

Argentinien-USA: Auslieferung, Art. 4 III (Indirekt: Doppelstaater)

Bulgarien-Polen: Doppelstaater

Polen-USA: Konuslar, Noten über Doppelstaater

Polen-USA: Wiedererwerb poln. StA durch in USA eingebürgerte Polen

BRD-DDR: Grundvertrag, Protokoll-Erklärungen 
$\begin{array}{lll}163) & 29.4 .(=) & \text { Indien-Sri Lanka: Kommuniqué, Ziff. } 14 \\ 164) & 10.10 .(31.3 .74) & \text { DDR-CSR: Doppelstaater } \\ 165) & \mathrm{x} 11.12 .(19.4 .74) & \text { BRD-CSR: Normalisierung, Art. II, } 2\end{array}$

1974

166) 29.1.(=)

167) 15.4.(28.5.75)

168) 31.5.(=)

169) 31.5.(4.5.75)

170) $\times$ 10.6.(1.12.77)

171) $\quad$ 4.7.(=)

172) $\mathrm{x}$ 10.9.(1.11.76)

1975

173) 15.2.(30.9.86)

173a) 29.3.(13.10.)

173b) $\times$ 23.5.(1.1.76)

173c) 11.9.(22.3.84)

174) $\quad 10.11 .(3.4 .77)$

175) $\times 12.11 .(25.4 .76)$

176) $25.11 .(=)$

1976

177) .April

178) 5.5.(=)

179) 2.6.(nie)

180) $\quad 14.7$.

1977

181) 6.5.(8.9.)

182) 1.6.(2.1.78)

183) 15.8.(25.2.78)

1978

183a) 20.6.(20.8.80)

184) 28.6.(16.6.79)

DDR-Mongolei: Doppelstaater

BRD-Tonga: Freundschaft, Art. 3

Mongolei-Ungarn: Doppelstaater

BRD-USA: Auslieferung, Art. 7 II (Einbürgerung)

Rumänien-SU: Doppelstaater
Indien-Sri Lanka: Kommuniqué, Absatz 21-22

Bulgarien-USA: Konsular, Noten über Doppelstaater

China-Malaysia: Kommunique, Punkt 5

Bulgarien-CSR: Doppelstaater

Italien-Spanien: WDS (dazu Noten v. 2. 11. 77)

Spanien-Venezuela: AEM

Frankreich-Italien: WDS
Nordmarianen-USA: Covenant, Sec. 301-304

DDR-Osterreich: Konsuln, Art. 1 II (Doppelstaater)

Mongolei-Polen: Doppelstaater

Mongolei-SU: Doppelstaater; mit Protokoll über Außerkrafttreten eines StA-Vertrages v. 24. 4. 1937

Italien-Jugoslawien: Osimo-Vertrag über Triest, Art. 3 u. Annex VI

DDR-Polen: Doppelstaater

Niederlande-Surinam: Verteilung der StA
Guinea Bissau-Senegal: Repatriierungs-Programm, Option

BRD-Großbritannien: Noten zu Konsularvertrag m. DDR v. 4. 5. 76

Mikronesien-USA: Covenant, Sec. 701-702

BRD-Italien: Noten, Wiederanwendung v. 10. 12. 38: AEM 
185) 7.1.(31.12.83) Brunei-Großbritannien: Noten betr. StA nach Unabhängigkeit

186) $\quad 31.1 .(=)$

187) 20.4.(17.2.80) China-USA: Annex über Doppelstaater zum Konsularagreement

188) 13.6.(24.2.80) DDR-Rumänien: Doppelstaater

189) $\quad 27.6(1.8 .80)$

Rumänien-Ungarn: Doppelstaater

190) $\quad 4.9 .(18.2 .81)$

Columbien-Spanien: Doppelstaater

191) $\quad$ 13.9.(1.2.81)

DDR-USA: Konsularvertrag, Art. 39 Z. 11 (Doppelstaater)

Argentinien-Osterreich: WDS

1980

191a) 6.6.(5.7.81)

192) $\quad$ 17.9.(19.2.82)

193) $\quad 28.10 .(5.2 .83)$

194) $\quad 31.10$.

195) $\quad 3.11 .(1.6 .84)$

1982

196) 18.3.(1.7.83)

197) 21.8.(13.9.)

1983

198) 29.11.(26.5.84) Bulgarien-Mongolei: Doppelstaater

1984

199) 9.7.(20.11.85)

200) $\quad 19.12 .(27.5 .85)$

1986

201)

15.1.(15.1.)
SU-CSSR: Doppelstaater

China-USA: Note betr. Doppelstaater zum Konsularvertrag

Italien-San Marino, Art. 39 bis in Freundschaftsvertrag v. 1939 eingefügt, Noten zur StA

USA mit Mikronesien, Palau u. Marshall-Inseln: Compact, Sec. 126

Belgien-Italien: WDS, mit Protokoll

Frankreich-Tunesien: WDS (dazu Noten v. 17.6. 82)

Neuseeland-Westsamoa: StA

Mongolei-CSSR: Doppelstaater

China-Großbritannien: Hong Kong, Memoranden über StA 